\title{
Multimodal hub location and hub network design
}

\author{
Sibel A. Alumur ${ }^{\mathrm{a}, *}$, Bahar Y. Kara ${ }^{\mathrm{b}}$, Oya E. Karasan ${ }^{\mathrm{b}}$ \\ a Department of Industrial Engineering, TOBB University of Economics and Technology, Sögütözü 06560, Ankara, Turkey \\ ${ }^{\mathrm{b}}$ Department of Industrial Engineering, Bilkent University, Bilkent 06800, Ankara, Turkey
}

\section{A R T I C L E I N F O}

\section{Article history:}

Received 1 March 2011

Accepted 23 February 2012

Processed by Associate Editor Yagiura

Available online 3 March 2012

Keywords:

Hub location

Network design

p-Hub median

Hub covering

\begin{abstract}
A B S T R A C T
Through observations from real life hub networks, we introduce the multimodal hub location and hub network design problem. We approach the hub location problem from a network design perspective. In addition to the location and allocation decisions, we also study the decision on how the hub networks with different possible transportation modes must be designed. In this multimodal hub location and hub network design problem, we jointly consider transportation costs and travel times, which are studied separately in most hub location problems presented in the literature. We allow different transportation modes between hubs and different types of service time promises between origindestination pairs while designing the hub network in the multimodal problem. We first propose a linear mixed integer programming model for this problem and then derive variants of the problem that might arise in certain applications. The models are enhanced via a set of effective valid inequalities and an efficient heuristic is developed. Computational analyses are presented on the various instances from the Turkish network and $\mathrm{CAB}$ data set.
\end{abstract}

(c) 2012 Elsevier Ltd. All rights reserved.

\section{Introduction}

Hub facilities are present in many transportation and telecommunication networks. In these networks, hubs usually act as sorting, transshipment, and consolidation points. Instead of sending flows directly between all origin-destination pairs, hub facilities consolidate flow in order to take advantage from the economies of scale.

Hub location problems arise in all network settings where there is a hub. The aim in these problems is to find the location of hub nodes and the allocation of demand nodes to these located hub nodes. The quadratic nature of the objective functions in hub location problems distinguishes them from the classical location problems. In standard location problems when the locations of the facilities are determined each demand node receives service from its nearest facility. For hub location problems, when it comes to allocation decisions, the nearest allocation strategy-assigning each demand node to its nearest hub-does not necessarily give optimal solutions. Thus the optimal allocations of demand centers to the located hubs must also be determined.

The interest in hub location grew with the pioneering works of O'Kelly [33,34]. The hub location literature is usually classified in terms of the objective function of the presented mathematical

\footnotetext{
* Corresponding author. Fax: +90 3122924091.

E-mail addresses: salumur@etu.edu.tr (S.A. Alumur), bkara@bilkent.edu.tr (B.Y. Kara), karasan@bilkent.edu.tr (O.E. Karasan).
}

models and the allocation structure. Single or multiple allocation hub location problems with total transportation cost objectives (median), min-max type objectives (center), and covering type constraints are well studied in the literature. The interested reader is referred to the reviews by Campbell et al. [11] and Alumur and Kara [1].

In most of the studies in the hub location literature, the hub network connecting the hub nodes is assumed to be complete with a presence of a direct hub link between every hub pair. In reality, many less-than truckload and telecommunication networks do not operate on a complete hub network structure $[19,29]$. There are few studies taking into account hub network design decisions in hub location problems. Perhaps, O'Kelly and Miller [36] was first to mention the possibility of using different hub network design protocols in the hub location literature. Nickel et al. [32] presented a new hub location problem arising in urban public transportation networks. They considered this hub location problem as a network design problem and incurred a fixed cost for locating hub links while minimizing the total transportation costs plus the fixed costs of locating hubs and building hub links. Podnar et al. [39] considered a new network design problem where they do not locate hubs but they decide on the links with reduced unit transportation costs. Yoon and Current [46] studied the multiple allocation hub location and hub network design problem with fixed and variable arc costs. They also considered direct connections between non-hub nodes and incurred variable arc costs associated with demand on the arcs. 
Campbell et al. [12,13] proposed hub arc location problems to the literature. Such problems locate hub arcs with reduced unit costs, rather than locating hub facilities. A fixed number of hub arcs is located while minimizing the total transportation costs. Campbell et al. [13] presented integer programming formulations for four special cases of the general multiple allocation hub arc location model and presented an enumeration-based algorithm. Campbell et al. [14] presented a parallel implementation of this algorithm in an attempt to solve larger hub arc location problems.

A recent study by Alumur et al. [3] introduced the single allocation incomplete hub network design problems to the literature. The authors studied median, center, and covering type single allocation hub location and hub network design problems and proposed efficient mathematical formulations to all versions of the problems. Alumur et al. [3] showed that building complete hub networks may result in some hub links carrying low amounts of flow yet employing economies of scale discount factor. In the models considering travel time, they showed that, in most of the instances, there is no need to establish a complete hub network to provide a required service quality. Calik et al. [8] considered the hub covering version of this network design problem. Alumur and Kara [2] worked on a special case where the hub network has a diameter of three. Another special case where the hub network is an undirected tree is introduced as the tree of hubs location problem to the literature by Contreras et al. [17,18].

Even though there is an inherent multicriteria nature underlying hub network design decisions arising in real life applications, the hub location literature customarily focuses on the single objective problems. In an ideal setting, the hub network design decisions should take into account both the transportation costs and the service quality. For example for cargo delivery sector, there is usually a given service time promise for origin-destination pairs. On the other hand, the cargo firms would like to minimize both the fixed and operating costs of establishing the hub network and the total costs of transportation for providing service within the promised service times $[7,42,43]$. A recent study by Campbell [10] addresses this deficiency in the hub location literature. In this paper, the author proposed time definite models for multiple allocation $p$-hub median and hub arc location problems. For both of the problems, a constraint is introduced on the maximum travel distance for each origindestination pair. Campbell [10] is the first study minimizing transportation costs subject to a constraint on the service level in hub location problems. A follow up study by Yaman et al. [45] also addresses this issue and proposes models incorporating transportation costs and service levels for complete hub networks.

Another important aspect in designing hub networks which has been traditionally overlooked is the choice for mode of transportation. It is assumed that there is only one hub type and one type of transportation mode in most of the hub location models presented in the literature. However, there is usually a choice between air, ground, and water transportation systems. For example, various cargo companies operating globally employ two different transportation modes, mainly air and ground transportation. There are various studies on the design of intermodal transportation networks. Reviews on intermodal transportation can be found in Bontekoning et al. [6] and Crainic and Kim [20]. Since we are concerned with locating hub facilities, we will focus on the studies considering hub location decisions in multimodal networks.

The concept of mode choice was first introduced to the hub location literature by O'Kelly and Lao [35]. In this study, O'Kelly and Lao consider two hubs (one master and one mini hub) at fixed locations and analyze the allocation decisions for air and ground transportation. More recently, there are some studies considering hub location decisions in intermodal networks. Arnold et al. [4] presented an integer programming model and a heuristic algorithm for locating rail/road terminals for freight transportation. Racunica and Wynter [40] used the uncapacitated hub location problem with a non-linear concave cost function accounting for the economies of scale to increase the share of rail in intermodal transportation. Groothedde et al. [25] focused on designing a hub based distribution network within a case study in the fast moving consumer goods market. Limbourg and Jourquin [28] determined the locations of rail-road transfer terminals using the single allocation $p$-hub median model. Ishfaq and Sox [26] extended the $p$-hub median model to include different modes of transportation, modal connectivity costs, and service time requirements. In a parallel study, Ishfaq and Sox [27] used uncapacitated hub location model with the inclusion of a service time constraint to solve for the location of hubs within a rail-road intermodal network. Meng and Wang [30] proposed a mathematical formulation to design an intermodal hub network for multi-type container transportation, which is suitable when there are multiple stakeholders such as the network planner, carriers, hub operators, and intermodal operators.

The inclusion of service level in the design phase brings along the consideration of different service levels for different types of customers. For example, most of the less-than truckload firms offer different delivery schedules for their customers such as overnight delivery and second day delivery, for different origindestination pairs. This issue has been considered in Yaman et al. [45], and Ishfaq and Sox [26,27] only for complete hub networks.

Even though the previous studies in the literature consider different types of hub facilities to be located or the inclusion of different service levels, none of them is general enough to support incomplete hub network design. The inclusion of hub network design decisions adds on extra modeling challenges and brings along the incorporation of operational costs for hub links.

In this paper, we introduce a problem that addresses the multicriteria nature of hub location problems by considering cost and service levels simultaneously, relaxes the complete hub network assumption, allows multiple modes of transportation, and considers different types of service levels for customers. To the best of the authors' knowledge, this is the most general form of the hub location network design problems addressed in the literature. This new problem decides on the location of different types of hubs, the allocation of the non-hub nodes to the located hubs, and which hub links to establish between hubs with which type of transportation mode. There are given service time parameters which may differ for each origin-destination pair. The aim is to minimize the total cost which is composed of the fixed costs of establishing the hub network and the total costs of transportation. We name the problem with these specifications as the multimodal hub location and hub network design problem.

The point of origin of this study is a small parcel delivery application arising in a single firm where the firm is responsible for the entire carriage. The firm operates its own fleet on the network connections and makes the crucial decisions on which links to operate its aircrafts and trucks.

The outline of this paper is as follows. In the next section, mathematical formulations for the most general case and a variant of the multimodal hub location and hub network design problem are introduced. In the third section, we present formulations for some of the classical hub location problems using the notation and constraints from the generic mathematical model that we propose. In the fourth section, we propose some valid inequalities for enhancing the model. The fifth section presents an application of the model on the Turkish network. In this section, we also present computational analyses with the valid inequalities, a heuristic algorithm for the problem, and solutions on the 
Turkish network and $C A B$ data set. The paper ends with some concluding remarks presented in the last section.

\section{Mathematical model}

In this section, we present mathematical formulations for the single allocation multimodal hub location and hub network design problem. The aim of the problem is to decide on the location of the hub nodes, the allocation of the non-hub nodes to these hub nodes, and which types of hub links to establish between the hub nodes. We aim to design our network so that the demand of each origin-destination pair receives service within the given service time bounds. The objective is to minimize the total costs which include the fixed costs of building and operating the hub network and the transportation costs. Initially, we provide a model for the most general case and subsequently, we introduce a more compact formulation with two types of hubs and hub links and two different service time promises motivated from the small parcel delivery applications.

\subsection{Generic model}

For the mathematical model, we need a given node set $N$ consisting of $n$ demand nodes and a potential hub set $H$ such that $H \subseteq N$ with $h$ nodes. There are different possible service types (denoted by set S). Additionally, there are different types of transportation modes (denoted by set $\mathrm{M}$ ). Different types of hubs and hub links can be established to facilitate these transportation modes. The unit transportation costs and travel times are dependent on the mode of transportation. It is assumed that different transportation modes are allowed to be used only within the hub network; that is, only for traveling between the hub nodes. For the allocation decisions, only ground transportation $(g \in M)$ is employed. There are no capacity restrictions. We assume throughout our model that the triangle inequality is satisfied for the parameters related to unit transportation cost and travel time within the same mode of transportation.

The parameters required for the mathematical model are listed as follows:

$w_{i j}^{s} \quad$ demand between nodes $i \in N$ and $j \in N$ for service type $s \in S$.

$c_{i j}^{m} \quad$ transportation cost of a unit of flow between nodes $i \in N$ and $j \in N$ using transportation mode $m \in M$.

$o c_{k}^{m} \quad$ unit operational cost at hub $k \in H$ with transportation mode $m \in M$.

$\mathrm{FH}_{k}^{m} \quad$ fixed cost of establishing and operating a hub at node $k \in H$ with transportation mode $m \in M$.

$F L_{k l}^{m} \quad$ fixed cost of operating a hub link between hubs $k \in H$ and $l \in H$ with transportation mode $m \in M$.

$t_{i j}^{m} \quad$ travel time between nodes $i \in N$ and $j \in N$ using transportation mode $m \in M$.

ot $t_{k}^{m} \quad$ operational time required at hub $k \in H$ with transportation mode $m \in M$.

$\beta^{s} \quad$ service time bound for service type $s \in S$.

$\alpha_{c}^{m} \quad$ hub-to-hub transportation cost discount factor using transportation mode $m \in M$.

$\alpha_{t}^{m} \quad$ hub-to-hub transportation time discount factor using transportation mode $m \in M$.

$p \quad$ total number of hubs to be established.

The decision variables of the mathematical model are

1 if node $i \in N$ is allocated to hub at node $k \in H$; 0 otherwise.
$H_{k}^{m} \quad 1$ if a hub is established at node $k \in H$ using transportation mode $m \in M$; 0 otherwise.

$Y_{i j k l}^{m s} \quad 1$ if the flow originated at node $i \in N$ destined to node $j \in N$ with service type $s \in S$ uses the hub link $\{k, l\}$ from hub $k \in H$ to hub $l \in H$ with transportation mode $m \in M$; 0 otherwise.

$Z_{k l}^{m} \quad 1$ if a hub link is established between hubs $k \in H$ and $l \in H$ using transportation mode $m \in M$; 0 otherwise.

$T_{i j}^{s} \quad$ discounted travel time from node $i \in H$ to node $j \in H$ for service type $s \in S$ on the designed hub network.

$C_{i j}^{S} \quad$ discounted unit transportation cost from node $i \in N$ to node $j \in N$ for service type $s \in S$ on the designed hub network.

The multimodal hub location and hub network design problem is modeled as

(P0)

Min. $\sum_{m \in M k \in H} \sum_{k} F H_{k}^{m} H_{k}^{m}+\sum_{m \in M k \in H l \in H: l>k} \sum_{l} F L_{k l}^{m} Z_{k l}^{m}+\sum_{s \in S i \in N j \in N} \sum_{i j} w_{i j}^{s}$

s.t. $\quad \sum_{k \in H} X_{i k}=1 \quad \forall i \in N$

$X_{i k} \leq X_{k k} \quad \forall i \in N, \quad k \in H$

$H_{k}^{m} \leq X_{k k} \quad \forall k \in H, \quad m \in M$

$\sum_{k \in H} X_{k k}=p$

$Z_{k l}^{m} \leq H_{k}^{m} \quad \forall k, l \in H: k<l, m \in M \backslash\{g\}$

$Z_{k l}^{m} \leq H_{l}^{m} \quad \forall k, l \in H: k<l, m \in M \backslash\{g\}$

$Z_{k l}^{g} \leq \sum_{m \in M} H_{k}^{m} \quad \forall k, l \in H: k<l$

$Z_{k l}^{g} \leq \sum_{m \in M} H_{l}^{m} \quad \forall k, l \in H: k<l$

$\sum_{m \in M l \in H: l \neq k} \sum_{i j k l}^{m s}-\sum_{m \in M l \in H: l \neq k} \sum_{i j l k}^{m s}=X_{i k}-X_{j k}$

$\forall i, j \in N: i \neq j, k \in H, s \in S$

$Y_{i j k l}^{m s}+Y_{i j l k}^{m s} \leq Z_{k l}^{m} \quad \forall i, j \in N: i \neq j, k, l \in H: k<l, s \in S$

$C_{i j}^{s}=\sum_{k \in H: k \neq i} c_{i k}^{g} X_{i k}+\sum_{m \in M k \in H l \in H: l \neq k} \sum_{k}\left(o c_{k}^{m}+\alpha_{c}^{m} c_{k l}^{m}+o c_{l}^{m}\right) Y_{i j k l}^{m s}$

$+\sum_{k \in H: k \neq j} c_{k j}^{g} X_{j k} \quad \forall i, j \in N: i \neq j, s \in S$

$\begin{aligned} T_{i j}^{s}= & \sum_{k \in H: k \neq i} t_{i k}^{g} X_{i k}+\sum_{m \in M k \in H l \in H: l \neq k} \sum_{k}\left(o t_{k}^{m}+\alpha_{t}^{m} t_{i j}^{m}+o t_{l}^{m}\right) Y_{i j k l}^{m s} \\ & +\sum_{k \in H: k \neq j} t_{k j}^{g} X_{j k} \quad \forall i, j \in N: i \neq j, s \in S\end{aligned}$

$T_{i j}^{s} \leq \beta^{s} \quad \forall i, j \in N, \quad s \in S$

$C_{i j}^{s} \geq 0 \quad \forall i, j \in N, \quad s \in S$

$T_{i j}^{S} \geq 0 \quad \forall i, j \in N, \quad s \in S$

$X_{i k} \in\{0,1\} \quad \forall i \in N, k \in H$

$H_{k}^{m} \in\{0,1\} \quad \forall k \in H, m \in M$

$Z_{k l}^{m} \in\{0,1\} \quad \forall k, l \in H: k<l, m \in M$ 
$Y_{i j k l}^{m s} \in\{0,1\} \quad \forall i, j \in N: i \neq j, k, l \in H: k \neq l, m \in M, s \in S$

In the objective function (1), the first term calculates the fixed costs of establishing and operating hubs, the second term calculates the fixed costs of operating hub links, and the last term calculates the total cost of transportation. While calculating the transportation costs, the demand to be routed between all pairs of nodes for different service types are multiplied with the $C_{i j}^{s}$ variables. The values of the $C_{i j}^{s}$ variables are calculated within the model using the hub network to be designed.

By constraints (2) and (3) every demand node is allocated to a hub node. These are the classical single allocation constraints in hub location. Constraint (4) allows establishing and operating different modes of transportation at a given hub node. By constraint (5), the model establishes $p$ hub nodes.

Constraints (6) and (7) ensure that a hub link of a certain transportation mode can only be established if both of the end nodes of that link are hubs established for that transportation mode. On the other hand, ground hub links can be established between all hub nodes via constraints (8) and (9). This distinction is because transportation modes other than ground transportation can only be utilized with specific terminals and/or equipment, whereas ground transportation is available between every hub.

Constraint (10) is the flow conservation constraint. This constraint determines which hub links to be used to route the demand for different service types between each origin-destination pair. Constraint (11) guarantees that the flow is routed only on the established hub links.

Constraint (12) calculates the discounted unit transportation cost between every node for each service type. Similarly, constraint (13) calculates the travel time between every node in the hub network for each service type. Constraint (14) ensures that the travel times between origin-destination pairs are less than the given time limit for each service type.

The rest of the constraints of the model are the non-negativity constraints and the constraints defining binary variables.

The proposed model (P0) allows operating different types of hubs and multiple modes of transportation. Especially in small parcel delivery, capacity is typically not an issue and strategic level decisions usually consider determining which segments to operate. Thus, at least in the design phase, at most one type of hub link can be considered for each segment.

One other key issue for such service networks is to clarify whether a VIP type service is feasible or not for certain origindestination pairs. In the strategic design phase, it is more crucial to design the network where different service types are promised to given set of customers. One may question the feasibility of a service network where only certain origin-destination pairs receive the VIP service. These assumptions also help in simplifying (P0) towards a more tractable $O\left(n^{3}\right)$ formulation which will be detailed in the next section.

\subsection{A more compact formulation with two transportation modes and two service time promises}

In this variant, we consider the problem with two types of hubs and hub links and two different service time promises. The developed model is readily extendible to more than two service types and transportation modes as long as the assumptions mentioned in the previous section are still valid.

More formally, there are two different types of transportation modes: air (denoted by $a$ ) and ground (denoted by $g$ ), and there are two types of hubs and hub links that can be established based on these two transportation modes. At most one type of hub link can be operated between two hub nodes. Additionally, there are two different types of service time promises: tight $\left(\beta_{1}\right)$ and loose $\left(\beta_{2}\right)$, such that $\beta_{1} \leq \beta_{2}$. A given set of nodes, say $N_{0} \subseteq N$ is to pairwise receive tight or VIP service. Let $S_{\beta_{1}}$ be the set of origindestination pairs of $N_{0}$ and $S_{\beta_{2}}$ be the remaining set of pairs to receive the loose service time bound.

Note that since we assume only one type of service between each origin-destination pair, there is no need for an index $s$ in the demand. Thus, $w_{i j}$ denotes the total demand between nodes $i \in N$ and $j \in N$.

It is possible to model this special case of the multimodal hub location and hub network design problem with $O\left(n^{3}\right)$ variables and $O\left(n^{3}\right)$ constraints. For this, we employ some ideas from the incomplete hub network design models introduced in Alumur et al. [3]. For each established hub, we find a spanning tree rooted at this hub that visits every other hub in the hub network using only the established hub links. The connectivity of the hub network is assured by employment of such spanning trees. We then calculate the travel time and the transportation costs between all pairs of hubs using these spanning trees. Since, there are two types of service time parameters we introduce two radii for each hub node to ensure that all origin-destination pairs receive service within their service time bounds. The radius of a hub can be defined as the maximum travel time from the non-hub nodes allocated to this hub to the hub node.

Additional decision variables required for the mathematical model are:

$Y_{i j k} \quad 1$ if the spanning tree rooted at hub $k \in H$ uses the hub link $\{i, j\}$ from hub $i \in H$ to hub $j \in H$; 0 otherwise.

$R_{j}^{1} \quad$ radius of hub $j \in H$ for tight service time bound.

$R_{j}^{2} \quad$ radius of hub $j \in H$ for loose service time bound.

Mathematical formulation for this variant of the multimodal hub location and hub network design problem is as follows:

$$
\begin{array}{ll}
\text { Min. } & \sum_{k \in H} F H_{k}^{a} H_{k}^{a}+\sum_{k \in H} F H_{k}^{g} H_{k}^{g}+\sum_{i \in H j \in H: j>i} \sum_{i j} F L_{i j}^{a} Z_{i j}^{a} \\
+ & \sum_{i \in H j \in H: j>i} \sum_{i j} F L_{i j}^{g} Z_{i j}^{g}+\sum_{i \in N j \in N} \sum_{i j} C_{i j}
\end{array}
$$

s.t. (2)-(9), (15)-(19)

$\sum_{i \in H: i \neq j} Y_{i j k} \geq X_{k k}+X_{j j}-1 \quad \forall j, k \in H: j \neq k$

$\sum_{i \in H: i \neq j} Y_{i j k} \leq X_{k k} \quad \forall j, k \in H: j \neq k$

$Y_{i j k}+Y_{j i k} \leq Z_{i j}^{a}+Z_{i j}^{g} \quad \forall i, j, k \in H: i<j$

$Z_{i j}^{a}+Z_{i j}^{g} \leq 1 \quad \forall i, j \in H: i<j$

$T_{k j} \geq\left(T_{k i}+\left(o t_{i}^{a}+\alpha_{t}^{a} t_{i j}^{a}+o t_{j}^{a}\right)\left(Z_{i j}^{a}+Z_{j i}^{a}\right)+\left(o t_{i}^{g}+\alpha_{t}^{g} t_{i j}^{g}+o t_{j}^{g}\right)\left(Z_{i j}^{g}+Z_{j i}^{g}\right)\right) Y_{i j k}$

$\forall i, j, k \in H: i \neq j, j \neq k$

$C_{k j} \geq\left(C_{k i}+\left(o c_{i}^{a}+\alpha_{c}^{a} c_{i j}^{a}+o c_{j}^{a}\right)\left(Z_{i j}^{a}+Z_{j i}^{a}\right)+\left(o c_{i}^{g}+\alpha_{c}^{g} C_{i j}^{g}+o c_{j}^{g}\right)\left(Z_{i j}^{g}+Z_{j i}^{g}\right)\right) Y_{i j k}$

$\forall i, j, k \in H: i \neq j, j \neq k$

$R_{j}^{1} \geq t_{i j}^{g} X_{i j}-M\left(1-X_{i j}\right) \quad \forall j \in H, i \in N: \exists k:(i, k) \in S_{\beta_{1}}$

$R_{j}^{1}+T_{j k}+R_{k}^{1} \leq \beta_{1} \quad \forall j, k \in H$

$R_{j}^{2} \geq t_{i j}^{g} X_{i j} \quad \forall i \in N, j \in H$ 
$R_{j}^{2}+T_{j k}+R_{k}^{2} \leq \beta_{2} \quad \forall j, k \in H$

$C_{i j} \geq\left(C_{i k}+c_{k j}^{g}\right) X_{j k} \quad \forall i, j \in N: i \neq j, k \in H$

$C_{i j}=C_{j i} \quad \forall i, j \in N$

$Y_{i j k} \in\{0,1\} \quad \forall i, j, k \in H: i \neq j, j \neq k$

In the objective function (21), the first two terms calculate the fixed costs of establishing and operating air and ground hubs; the next two terms calculate the fixed costs of operating hub links with air and ground transportation modes. The last term in the objective function calculates the total cost of transportation.

Constraints (22) and (23) establish the rooted spanning trees. Constraint (24) guarantees that the spanning trees can only use the established hub links. By constraint (25), only one type of hub link can be established between two hub nodes.

Constraint (26) calculates the discounted travel time between every hub node in the hub network using the spanning trees. Similarly constraint (27) calculates the discounted unit transportation cost between every hub node in the hub network using the spanning trees.

We defined two radii for each hub node to be established. The first radius of a hub node calculates the maximum travel time among all non-hub nodes that are allocated to this hub node and which are an origin requiring service within $\beta_{1}$ to any destination node. Constraint (28) calculates the first radius of a hub. It suffices to calculate the first radius of a hub node using only the origins of the set $S_{\beta_{1}}$, since it is assumed that service levels are symmetric for origin-destination pairs. If there is no node allocated to a hub node requiring service within $\beta_{1}$, then the tight radius associated with that hub node is bounded below by a negative number. It suffices to define $M$ as $\beta_{2}-\beta_{1}+\max _{i \in N, j \in H} t_{i j}^{g}$. Constraint (29) ensures that the travel times between origin-destination pairs requiring service within $\beta_{1}$ are satisfied.

The second radius is calculated in Constraint (30) as the maximum travel time from a non-hub node to its allocated hub. We ensure by Constraint (31) that the travel time between any two nodes in the network is less than $\beta_{2}$ because all origindestination pairs must either receive service within $\beta_{1}$ or $\beta_{2}$, and $\beta_{1} \leq \beta_{2}$.

Note that by the employment of the radii variables, $R_{j}^{1}$ and $R_{j}^{2}$, it is sufficient to calculate the travel time only between the hub nodes.

The discounted unit transportation costs are calculated within all pairs of hub nodes in constraint (27). Constraints (32) and (33) calculate the unit transportation costs between all pairs of nonhub nodes in the network. We assumed that the flow costs are symmetric. However, replacement of constraint (33) with $C_{i j} \geq\left(c_{i k}^{g}+C_{k j}\right) X_{i k}$ handles the non-symmetric case.

This is a non-linear programming model due to constraints (26), (27), and (32). In the sequel, we provide tight Big- $M$ type linearizations for these non-linear constraints.

First, we propose the following constraint for the linearization of constraint (26):

$$
\begin{gathered}
T_{k j} \geq T_{k i}+\left(o t_{i}^{a}+\alpha_{t}^{a} t_{i j}^{a}+o t_{j}^{a}\right)\left(Z_{i j}^{a}+Z_{j i}^{a}\right)+\left(o t_{i}^{g}+\alpha_{t}^{g} t_{i j}^{g}+o t_{j}^{g}\right)\left(Z_{i j}^{g}+Z_{j i}^{g}\right) \\
-M_{1}\left(1-Y_{i j k}\right) \quad \forall i, j, k \in H: i \neq j, j \neq k
\end{gathered}
$$

where $M_{1}=\beta_{2}+\max _{i, j \in H}\left(o t_{i}^{a}+\alpha_{t}^{a} t_{i j}^{a}+o t_{j}^{a}, o t_{i}^{g}+\alpha_{t}^{g} t_{i j}^{g}+o t_{j}^{g}\right)$.

For the linearizations of constraints (28) and (33), let $E$ be the set of hub links that can be established. More formally,

$$
\begin{aligned}
E= & \{e=\{i, j\}: i, j \in H: i<j\}=\left\{e_{1}, e_{2}, \ldots, e_{h(h-1) / 2}\right\} \\
& \text { We define } \\
\gamma_{e}= & \max \left\{o c_{i}^{a}+\alpha_{c}^{a} c_{i j}^{a}+o c_{j}^{a}, o c_{i}^{g}+\alpha_{c}^{g} c_{i j}^{g}+o c_{j}^{g}\right\} \quad \forall e=\{i, j\} \in E
\end{aligned}
$$

Assume without loss of generality that

$\gamma_{e_{1}} \geq \gamma_{e_{2}} \geq \cdots \geq \gamma_{e_{h(h-1) / 2}}$

We propose the following constraint for the linearization of constraint (27):

$$
\begin{aligned}
& C_{k j} \geq C_{k i}+\left(o c_{i}^{a}+\alpha_{c}^{a} c_{i j}^{a}+o c_{j}^{a}\right)\left(Z_{i j}^{a}+Z_{j i}^{a}\right)+\left(o c_{i}^{g}+\alpha_{c}^{g} c_{i j}^{g}+o c_{j}^{g}\right)\left(Z_{i j}^{g}+Z_{j i}^{g}\right) \\
& \quad-M_{2}\left(1-Y_{i j k}\right) \\
& \quad \forall i, j, k \in H: i \neq j, j \neq k
\end{aligned}
$$

where $M_{2}=2 \gamma_{e_{1}}+\gamma_{e_{2}}+\cdots+\gamma_{e_{(p-1)}}$.

Lastly, we propose the following constraint for the linearization of constraint (32):

$C_{i j} \geq C_{i k}+c_{k j}^{g} X_{j k}-M_{3}\left(1-X_{j k}\right) \quad \forall i, j \in N: i \neq j, k \in H$

where $M_{3}=\max _{i \in N, j \in H}\left(c_{i j}^{g}\right)+\gamma_{e_{1}}+\gamma_{e_{2}}+\cdots+\gamma_{e_{(p-1)}}$.

A linear integer programming formulation of this special case of the multimodal hub location and hub network design problem consists of the objective function (21) and constraints (2)-(9), (15)-(19), (22)-(25), (26*), (27*), (28)-(31), (32*), (33) and (34). In the worst case $h=n$ and the linear integer programming formulation of this variant of the problem has $\mathrm{O}\left(n^{3}\right)$ variables and $\mathrm{O}\left(n^{3}\right)$ constraints.

\section{Formulations for some other hub location problems}

The multimodal hub location and hub network design problem incorporates many of the aspects of the real life applications such as incomplete hub network, multiple modes of transportation, multiple service levels, and multicriteria nature of cost and service quality. In its most general sense, (P0) answers all these requirements simultaneously. In different real life situations, different combinations of the above listed aspects may appear. Some of these problems at least conceptually appear in the existing hub location studies. However, most of them though quite realistic in nature have not been even defined in the literature. Perhaps, due to the challenging quadratic nature of the most basic form of hub location models, the literature have built and expanded on simplifying assumptions such as complete hub network, single mode of transportation, and single objective. The point of origin of this study is to tackle more real life situations by relaxing all these assumptions. Multimodal hub location and hub network design problem and the resulting model (P0) answer this purpose.

In its most general form, the defined problem might be more demanding than the particular application calls for. Some real life applications might only require a subset of the aspects existing in (P0) as in the case of model (P1). In the sequel, we show this unifying property of (P0) by introducing several additional problems and models to the hub location literature by focusing on different combinations of the mentioned real life aspects. In this way, we contribute to the literature by defining and modeling new hub location network design problems.

If there are no service level requirements in applications where cost is the primary issue, the multicriteria nature of our problem can be overlooked. We define the problem with only the transportation cost objective, the multimodal p-hub median network design problem, as follows:

(P2)Min. $\sum_{i \in N j \in N} \sum_{i j} C_{i j}$

s.t. (2)-(12), (15), (17)-(20)

In this formulation, objective function (35) is the last term in the objective function (1) of (P0) that accounts for the total transportation costs with a single service type. We simply discard the constraints related to travel times from (P0) to obtain the 
constraints for the multimodal $p$-hub median network design problem.

If one sets $Z_{i j}^{m}=0 \forall i, j \in H, m \in M \backslash\{g\}$ and $H_{j}^{m}=0 \forall j \in H, m \in$ $M \backslash\{g\}$ in the above multimodal $p$-hub median network design formulation, then the problem reduces to the incomplete $p$-hub median problem with single mode of transportation introduced in Alumur et al. [3].

In certain applications where the strategic decisions are dominant, the transportation costs (which are usually considered as operational costs) might be secondary. In such situations, the main concern is whether a feasible design meeting certain service level requirements exists or not and this naturally triggers the covering objectives. Our multimodal hub location and hub network design model readily includes multiple/single mode hub covering network design problems with different service time promises. We introduce the mathematical formulation for the multimodal hub covering network design problem with different service time promises:

(P3)

$$
\text { Min. } \sum_{m \in M} \sum_{k \in H} F H_{k}^{m} H_{k}^{m}+\sum_{m \in M} \sum_{k \in H l \in H: l>k} \sum_{k l} L_{k l}^{m} Z_{k}^{m}
$$

s.t. (2)-(4), (6)-(11), (13), (14), (16)-(20)

In this formulation, we have excluded the transportation costs from the objective function and the constraints related to transportation costs from (P0).

Similar to the $p$-hub median version, if we have a single service type and if we set $Z_{i j}^{m}=0 \forall i, j \in H, m \in M \backslash\{g\}$ and $H_{j}^{m}=0 \forall j \in H$, $m \in M \backslash\{g\}$ in the above formulation, then the problem reduces to the incomplete hub covering problem with single mode of transportation studied in Alumur et al. [3] and Calik et al. [8].

In addition to the previously presented models, it is also possible to model the classical single allocation $p$-hub median problem introduced by O'Kelly [34] and the classical hub covering problem introduced by Campbell [9] with complete hub networks by using the multimodal hub location and hub network design formulation. In this section, apart from the problem under the scope of this study, we have introduced additional hub network design problems with realistic features, namely, the multimodal p-hub median network design problem (corresponding model P2), the multimodal hub covering network design problem with single and multiple (corresponding model P3) service time promises to the literature. All of these new problems are generic in nature, and different variants of them can be derived for specific applications. Moreover, the mathematical models for all of these problems can also be derived from (P1) when the underlying assumptions are valid.

\section{Valid inequalities}

In this section, we derive several families of valid inequalities. For simplicity in presentation, the forthcoming valid inequality derivations are presented for (P1). However, they can easily be extended to the feasible region of (P0). The methodology borrows ideas from Yaman et al. [45]. The proofs of the validity of all the inequalities are presented in the Appendix.

Let $F$ be the feasible set for (P1). That is, $F$ is the set of solutions satisfying the constraints (2)-(9), (15)-(19), (22)-(25), (26*), $\left(27^{*}\right),(28)-(31),\left(32^{*}\right),(33)$ and (34).

As already stated before, it is assumed that triangle inequality is satisfied for both the unit transportation costs and the travel times for the mathematical model. For the sets of valid inequalities to be introduced, we additionally assume that $\alpha_{c}^{g} c_{i j}^{g} \leq \alpha_{c}^{a} c_{i j}^{a}$ and $\alpha_{t}^{a} t_{i j}^{a} \leq \alpha_{t}^{g} t_{i j}^{g} \forall i, j \in N$. That is, the unit discounted transportation cost using air transportation is assumed to be higher than the unit discounted transportation cost of using ground transportation between any two nodes, and the discounted travel time using air transportation is assumed to be lower than the discounted travel time using ground transportation between any two nodes. We expect the unit transportation costs associated with ground transportation to be lower than the corresponding unit transportation costs of using air transportation $c_{i j}^{g} \leq c_{i j}^{a} \forall i, j \in N$ and the travel times using air transportation to be lower than the travel times using ground transportation $t_{i j}^{a} \leq t_{i j}^{g} \forall i, j \in N$. We observed from cargo applications that the unit transportation costs of using air transportation is order of magnitudes higher than that of using ground transportation. Similarly, the transportation times of using air transportation is order of magnitudes lower than that of using ground transportation. Thus, the assumptions of having $\alpha_{c}^{g} c_{i j}^{g} \leq \alpha_{c}^{a} c_{i j}^{a}$ and $\alpha_{t}^{a} t_{i j}^{a} \leq \alpha_{t}^{g} t_{i j}^{g} \quad \forall i, j \in N$ are reasonable.

We first define some new parameters required for the definition of our valid inequalities. For the sake of simplicity, the unit operational costs and operational times at hubs are assumed to be embedded in the respective cost and time parameters. For $i \in N$ and $j \in N \backslash\{i\}$, let

$\lambda_{i j}^{1}=\min _{k \in H \backslash j\}}\left(\alpha_{c}^{g} c_{i k}^{g}+c_{k j}^{g}\right)$

$\lambda_{i j}^{2}=\min _{k \in H \backslash i\}}\left(c_{i k}^{g}+\alpha_{c}^{g} c_{k j}^{g}\right)$

$\lambda_{i j}^{3}=\min _{k \in H \backslash i i, j\}} \min _{l \in H \backslash\{i, j\}}\left(c_{i k}^{g}+\alpha_{c}^{g} c_{k l}^{g}+c_{l j}^{g}\right)$

Since triangle inequality holds for unit transportation costs and $\alpha_{c}^{g} c_{i j}^{g} \leq \alpha_{c}^{a} c_{i j}^{a} \forall i, j \in N$ by assumption, if node $i$ is a hub and node $j$ is not a hub, then the minimum unit transportation cost from node $i$ to node $j$ is $\lambda_{i j}^{1}$. (Note that, since node $k$ may be equal to node $i$ in the minimum operator, the case when node $j$ is allocated to hub $i$ is also covered.) Conversely, if node $j$ is a hub and node $i$ is not a hub, then $\lambda_{i j}^{2}$ is a lower bound on the unit transportation cost from node $i$ to node $j$. Finally, $\lambda_{i j}^{3}$ provides a lower bound on the unit transportation cost from node $i$ to node $j$ if neither of them are hub nodes.

Proposition 1. For $i \in H$ and $j \in H \backslash\{i\}$, inequalities

$C_{i j} \geq \alpha_{c}^{g} C_{i j}^{g}+\left(\lambda_{i j}^{1}-\alpha_{c}^{g} C_{i j}^{g}\right)\left(1-X_{j j}\right)+\min \left\{\lambda_{i j}^{3}-\lambda_{i j}^{1}, \lambda_{i j}^{2}-\alpha_{c}^{g} C_{i j}^{g}\right\}\left(1-X_{i i}\right)$

and

$C_{i j} \geq \lambda_{i j}^{3}+\left(\lambda_{i j}^{2}-\lambda_{i j}^{3}\right) X_{j j}+\min \left\{\lambda_{i j}^{1}-\lambda_{i j}^{3}, \alpha_{c}^{g} C_{i j}^{g}-\lambda_{i j}^{2}\right\} X_{i i}$

are valid for $F$.

For $i \in H$ and $j \in N \backslash H$, the inequality.

$C_{i j} \geq \lambda_{i j}^{1}+\left(\lambda_{i j}^{3}-\lambda_{i j}^{1}\right)\left(1-X_{i i}\right)$

is valid for $F$.

For $i \in N \backslash H$ and $j \in H$, the inequality.

$C_{i j} \geq \lambda_{i j}^{2}+\left(\lambda_{i j}^{3}-\lambda_{i j}^{2}\right)\left(1-X_{j j}\right)$

is valid for $F$.

For $i \in N \backslash H$ and $j \in N \backslash H$, the inequality.

$C_{i j} \geq \lambda_{i j}^{3}$

is valid for $F$.

The inequalities (A.1)-(A.5) are derived based on the information that a node becomes a hub or not, to obtain lower bounds on the unit transportation costs. In the next set of valid inequalities, we again obtain lower bounds for the unit transportation costs but this time use the information that if a node is not a hub then it must be allocated to a hub node. 
Proposition 2. For $i \in H$ and $j \in N \backslash\{i\}$, the inequality

$C_{i j} \geq \sum_{h \in H \backslash\{i\}}\left(c_{i h}^{g}+\min _{k \in H \backslash i i\}}\left(\alpha_{c}^{g} c_{h k}^{g}+c_{k j}^{g}\right)\right) X_{i h}+\min _{k \in H}\left(\alpha_{c}^{g} c_{i k}^{g}+c_{k j}^{g}\right) X_{i i}$

is valid for $F$.

For $i \in N \backslash H$ and $j \in N \backslash\{i\}$, the inequality.

$C_{i j} \geq \sum_{h \in H}\left(c_{i h}^{g}+\min _{k \in H}\left(\alpha_{c}^{g} c_{h k}^{g}+c_{k j}^{g}\right)\right) X_{i h}$

is valid for $F$.

For $i \in N$ and $j \in H \backslash\{i\}$, the inequality

$C_{i j} \geq \sum_{h \in H \backslash j\}}\left(\min _{k \in H \backslash j\}}\left(c_{i k}^{g}+\alpha_{c}^{g} c_{k h}^{g}\right)+c_{h j}^{g}\right) X_{j h}+\min _{k \in H}\left(c_{i k}^{g}+\alpha_{c}^{g} c_{k j}^{g}\right) X_{j j}$

is valid for $F$.

For $i \in N$ and $j \in N \backslash(H \cup\{i\})$, the inequality

$C_{i j} \geq \sum_{h \in H}\left(\min _{k \in H}\left(c_{i k}^{g}+\alpha_{c}^{g} c_{k h}^{g}\right)+c_{h j}^{g}\right) X_{j h}$

is valid for $F$.

Previous sets of valid inequalities, (A) and (B), are both derived to obtain lower bounds for the unit transportation costs between nodes. In the sequel, we introduce the valid inequalities related to travel time. For $i \in N$ and $j \in N \backslash\{i\}$, let

$\mu_{i j}^{1}=\min _{k \in H \backslash j\}}\left(\alpha_{t}^{a} t_{i k}^{a}+t_{k j}^{g}\right)$

$\mu_{i j}^{2}=\min _{k \in H \backslash i i}\left(t_{i k}^{g}+\alpha_{t}^{a} t_{k j}^{a}\right)$

$\mu_{i j}^{3}=\min _{k \in H \backslash\{i, j\}} \min _{l \in H \backslash i, j\}}\left(t_{i k}^{g}+\alpha_{t}^{a} t_{k l}^{a}+t_{l j}^{g}\right)$

Since triangle inequality holds for travel times and $\alpha_{t}^{a} t_{i j}^{a} \leq \alpha_{t}^{g} t_{i j}^{g} \forall i, j \in H$ by assumption, if node $i$ is a hub and node $j$ is not a hub, then the minimum travel time from node $i$ to node $j$ is $\mu_{i j}^{1}$. Conversely, if node $j$ is a hub and node $i$ is not a hub, then $\mu_{i j}^{2}$ is a lower bound on the travel time from node $i$ to node $j$. Finally, $\mu_{i j}^{3}$ provides a lower bound on the travel time from node $i$ to node $j$ if none of these nodes is a hub.

Proposition 3. For $i \in H$ and $j \in H \backslash\{i\}$, inequalities

$$
\begin{aligned}
& T_{i j} \geq \alpha_{t}^{a} t_{i j}^{a}+\left(\mu_{i j}^{1}-\alpha_{t}^{a} t_{i j}^{a}\right)\left(1-X_{j j}\right)+\min \left\{\mu_{i j}^{3}-\mu_{i j}^{1}, \mu_{i j}^{2}-\alpha_{t}^{a} t_{i j}^{a}\right\}\left(1-X_{i i}\right) \\
& T_{i j} \geq \mu_{i j}^{3}+\left(\mu_{i j}^{2}-\mu_{i j}^{3}\right) X_{j j}+\min \left\{\mu_{i j}^{1}-\mu_{i j}^{3}, \alpha_{t}^{a} t_{i j}^{a}-\mu_{i j}^{2}\right\} X_{i i} \\
& T_{i j} \geq \sum_{h \in H \backslash i i\}}\left(t_{i h}^{g}+\min _{k \in H \backslash i i\}}\left(\alpha_{t}^{a} t_{h k}^{a}+t_{k j}^{g}\right)\right) X_{i h}+\min _{k \in H}\left(\alpha_{t}^{a} t_{i k}^{a}+t_{k j}^{g}\right) X_{i i}
\end{aligned}
$$

$T_{i j} \geq \sum_{h \in H \backslash j\}}\left(\min _{k \in H \backslash j\}}\left(t_{i k}^{g}+\alpha_{t}^{a} t_{k h}^{a}\right)+t_{h j}^{g}\right) X_{j h}+\min _{k \in H}\left(t_{i k}^{g}+\alpha_{t}^{a} t_{k j}^{a}\right) X_{j j}$

are valid for $F$.

We tested the performance of these three sets of valid inequalities, (A), (B), and (C), both individually and collectively. Detailed results are provided in Section 5.1.

\section{Application in Turkey}

We applied our multimodal hub location and hub network design model (P1) on the Turkish network. Turkish network data set is used in various hub location studies [3,8,41,44] and is available in OR Library [5]. There are 81 demand nodes in this data set corresponding to 81 administrative districts of Turkey. Among these 81 nodes, the most populated and industrialized 16 cities are listed as the candidate set of hub locations in Yaman et al. [44] $(n=81, h=16)$. Fig. 1 shows the locations and the corresponding node numbers of the 81 demand centers and 16 candidate hub locations on a map of Turkey.

For the Turkish network, the values of the parameters $w_{i j}$, FH $F L_{i j}^{g}$, and $c_{i j}^{g}$, comply with the values presented in Beasley [5], where $c_{i j}^{g}$ is taken to be equal to the travel distances. To determine the value of the economies of scale parameter associated with ground transportation costs, several interviews are held with representatives from different cargo companies operating in Turkey. Based on these interviews $\alpha_{c}^{g}$ is taken as 0.8. We observed that the cost values associated with air hub and hub link usage is higher than that of ground hub and hub link usage, as expected. The costs of operating air hubs and air hub links are taken to be 10 times the corresponding values of using ground transportation. Since we could not find approximate values for the unit transportation costs using air transportation, we let $c_{i j}^{a}=r c_{i j}^{g} \forall i, j \in H$ and varied the value of $r$ in our experiments within the set $\{2,4,6,8\}$. We assumed that the economies of scale is reflected within these costs.

Travel times using ground transportation are calculated assuming a travel speed of $80 \mathrm{~km} / \mathrm{h}$ whereas travel times for using air transportation are estimated by assuming that the airplanes travel at a speed of $700 \mathrm{~km} / \mathrm{h}$. Since ground transportation can be used on the allocation links as well as on the hub links, we take $\alpha_{t}^{g}=0.9$ as suggested by Tan and Kara [41]. On the other hand, since air transportation is available only between hub nodes, the air travel times correspond to their respective discounted values. Moreover, the unit operational costs and operational times are assumed to be embedded in the respective cost and time parameters.

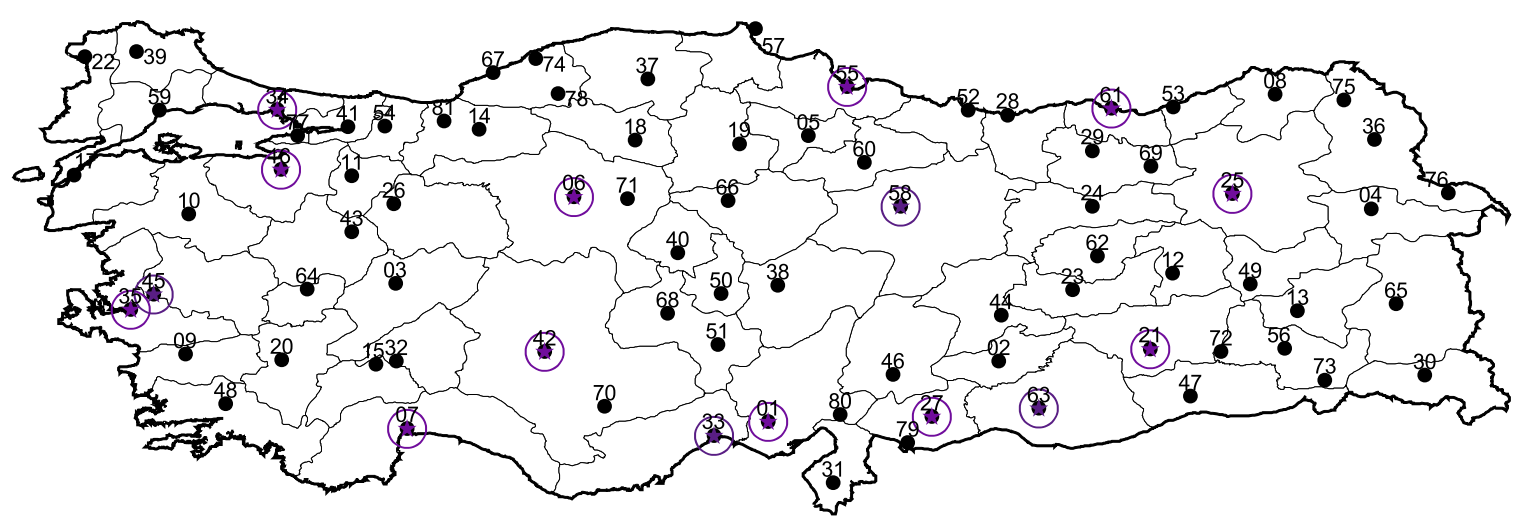

Fig. 1. The 81 demand nodes and the 16 candidate hub locations on the Turkish network. 
We took the service time bounds, as $\beta_{1}=12$ and $\beta_{2}=24 \mathrm{~h}$, where the first one corresponds to VIP service and the second to next day delivery, respectively. The set of origin-destination pairs requiring service within $12 \mathrm{~h}$ is taken as the same as the VIP service set of a well-known cargo company operating in Turkey. Based on their service promises, the demand centers Adana (1), Ankara (6), Antalya (7), Bursa (16), Erzurum (25), Istanbul (34), and Izmir (35) can mutually use the VIP service. So, all of these demand centers are mutually origins and destinations to each other in the set $S_{\beta_{1}}$. All of the remaining pairs from 81 demand centers are included in the set $S_{\beta_{2}}$.

In order to analyze the effect of aircraft fleet size on the resulting hub networks we define a new parameter $q$ and set the number of air hub links to be opened to $q$ within our computational analysis.

We varied the values of the remaining parameters $p, q$, and $r$. We took our runs on a server with $2.66 \mathrm{GHz}$ Intel Xeon processor and $8 \mathrm{~GB}$ of RAM and we used the optimization software Gurobi version 4.5.2.

In the next subsection, we present computational analysis with the valid inequalities introduced in the previous section of the paper. In Section 5.2, we introduce a simple heuristic for the problem and test its performance on the Turkish network and the CAB data set. Lastly, in Section 5.3, we report the solutions from the Turkish network data set.

\subsection{Computational analysis with valid inequalities}

For this analysis, we needed a smaller data set because the results with the 81 node Turkish network turned out to be inconclusive in reasonable times. Thus, we generated a smaller data set from the Turkish network. We took 25 nodes from 81 demand centers and chose 8 candidate hub locations from 16 presented in the Turkish network $(n=25, h=8)$. With this smaller Turkish data set we ranged the values of $p, q$, and $r$.

We tested and compared the three sets of valid inequalities, (A), (B), and (C), introduced in the fourth section, with (P1). We compared the initial lower bound values reported by Gurobi, the CPU time requirements, and the number of nodes in the branch and bound tree. We provide the results in Table 1 .

The first three columns in Table 1 report the instance parameters: number of hubs $(p)$, number of air hub links $(q)$, the ratio of the unit cost of using air transportation to ground transportation $(r)$, respectively. For each instance, the first row of column five lists the initial lower bound value reported by Gurobi (denoted by 'lb'), the second row lists the CPU time requirement in seconds by Gurobi (denoted by 'cpu'), and the last row lists the number of nodes in the branch and bound tree (denoted by 'nodes') reported by Gurobi. Columns 5-12 correspond to all possible combinations of the three sets of valid inequalities. In particular, the column indicated by 'No v.i.'s', corresponds to the

Table 1

The effect of valid inequalities.

\begin{tabular}{|c|c|c|c|c|c|c|c|c|c|c|c|}
\hline $\boldsymbol{p}$ & $q$ & $\boldsymbol{r}$ & Statistics & No v.i.'s & A & B & C & AB & $\mathrm{AC}$ & BC & $\mathrm{ABC}$ \\
\hline \multirow[t]{3}{*}{4} & 2 & 2 & $\mathbf{l b}$ & 2976.15 & 3234.67 & 3278.16 & 2976.15 & 3278.50 & 3234.67 & 3278.16 & 3278.50 \\
\hline & & & cpu & 352.99 & 14.12 & 10.55 & 257.71 & 29.00 & 16.65 & 9.78 & 15.12 \\
\hline & & & nodes & 487,402 & 5675 & 2727 & 173,637 & 3047 & 6113 & 2098 & 2267 \\
\hline \multirow[t]{3}{*}{4} & 2 & 4 & lb & 2976.15 & 3234.67 & 3278.16 & 2976.15 & 3278.50 & 3234.67 & 3278.16 & 3278.50 \\
\hline & & & cpu & 129.94 & 23.25 & 11.24 & 113.01 & 6.99 & 19.72 & 13.11 & 11.97 \\
\hline & & & nodes & 81,217 & 5659 & 3167 & 106,582 & 2145 & 10,547 & 1969 & 2448 \\
\hline \multirow[t]{3}{*}{4} & 2 & 6 & $\mathbf{l b}$ & 2976.15 & 3234.67 & 3278.16 & 2976.15 & 3278.50 & 3234.67 & 3278.16 & 3278.50 \\
\hline & & & cpu & 20.66 & 10.09 & 9.91 & 66.93 & 10.66 & 13.22 & 9.42 & 14.57 \\
\hline & & & nodes & 27,220 & 3102 & 2319 & 85,729 & 2431 & 3328 & 2465 & 3012 \\
\hline \multirow[t]{3}{*}{4} & 2 & 8 & lb & 2976.15 & 3234.67 & 3278.16 & 2976.15 & 3278.50 & 3234.67 & 3278.16 & 3278.50 \\
\hline & & & cpu & 27.13 & 10.12 & 10.50 & 16.22 & 12.99 & 14.11 & 12.75 & 13.42 \\
\hline & & & nodes & 32,080 & 3204 & 3032 & 15,051 & 2476 & 4863 & 2380 & 2856 \\
\hline \multirow[t]{3}{*}{4} & 3 & 2 & lb & 3884.95 & 4143.26 & 4186.70 & 3884.95 & 4187.07 & 4143.26 & 4186.70 & 4187.07 \\
\hline & & & cpu & 43.34 & 18.25 & 7.24 & 55.80 & 23.25 & 10.06 & 9.29 & 9.31 \\
\hline & & & nodes & 66,460 & 19,504 & 2690 & 61,499 & 3017 & 3565 & 2765 & 2795 \\
\hline \multirow[t]{3}{*}{4} & 3 & 4 & lb & 3884.95 & 4143.26 & 4186.70 & 3884.95 & 4187.07 & 4143.26 & 4186.70 & 4187.07 \\
\hline & & & cpu & 33.38 & 14.55 & 10.16 & 169.99 & 14.24 & 14.30 & 8.41 & 12.68 \\
\hline & & & nodes & 56,005 & 5525 & 2417 & 94,165 & 3573 & 5249 & 3453 & 3131 \\
\hline \multirow[t]{3}{*}{4} & 3 & 6 & lb & 3884.95 & 4143.26 & 4186.70 & 3884.95 & 4187.07 & 4143.26 & 4186.70 & 4187.07 \\
\hline & & & cpu & 21.24 & 16.77 & 10.00 & 22.31 & 11.53 & 13.08 & 16.10 & 13.64 \\
\hline & & & nodes & 24,520 & 10,572 & 3050 & 23140 & 3029 & 4088 & 5264 & 2820 \\
\hline \multirow[t]{3}{*}{4} & 3 & 8 & lb & 3884.95 & 4143.26 & 4186.70 & 3884.95 & 4187.07 & 4143.26 & 4186.70 & 4187.07 \\
\hline & & & cpu & 21.76 & 13.44 & 10.78 & 23.13 & 10.24 & 12.71 & 10.36 & 18.11 \\
\hline & & & nodes & 21,560 & 5076 & 2637 & 13,626 & 3232 & 2756 & 2748 & 2769 \\
\hline \multirow[t]{3}{*}{4} & 4 & 2 & lb & 4793.76 & 5051.85 & 5095.30 & 4793.76 & 5095.72 & 5051.85 & 5095.30 & 5095.72 \\
\hline & & & cpu & 698.73 & 36.28 & 17.37 & 1003.00 & 15.25 & 51.62 & 18.01 & 15.19 \\
\hline & & & nodes & 576,762 & 39722 & 6837 & 833,480 & 6385 & 47,117 & 5761 & 6360 \\
\hline \multirow[t]{3}{*}{4} & 4 & 4 & lb & 4793.76 & 5051.85 & 5095.30 & 4793.76 & 5095.72 & 5051.85 & 5095.30 & 5095.72 \\
\hline & & & cpu & 163.58 & 20.39 & 13.95 & 159.15 & 16.87 & 28.06 & 18.09 & 15.95 \\
\hline & & & nodes & 125,339 & 16,798 & 7039 & 213,272 & 9392 & 17,337 & 5662 & 6741 \\
\hline \multirow[t]{3}{*}{4} & 4 & 6 & lb & 4793.76 & 5051.85 & 5095.30 & 4793.76 & 5095.72 & 5051.85 & 5095.30 & 5095.72 \\
\hline & & & cpu & 51.38 & 23.08 & 14.70 & 35.40 & 14.98 & 24.09 & 12.92 & 16.29 \\
\hline & & & nodes & 87,191 & 22,510 & 6849 & 45,214 & 6003 & 15,385 & 5333 & 5544 \\
\hline \multirow[t]{3}{*}{4} & 4 & 8 & lb & 4793.76 & 5051.85 & 5095.30 & 4793.76 & 5095.72 & 5051.85 & 5095.30 & 5095.72 \\
\hline & & & cpu & 25.39 & 33.48 & 12.81 & 24.96 & 14.60 & 23.52 & 16.42 & 19.75 \\
\hline & & & nodes & 37,498 & 37,159 & 4554 & 28,016 & 5439 & 16,819 & 4436 & 5357 \\
\hline
\end{tabular}


linear integer formulation (P1) without any of the valid inequalities, and the last column includes all the three sets of valid inequalities in our formulation.

When we compare the initial lower bounds, we observe that inequality set (C) does not have any effect on the lower bounds. This is because these sets of inequalities are related to travel times which do not have associated costs in the objective function. The inequality set (B) always resulted in higher lower bounds when compared with set (A). Observe from Table 1 that the highest initial lower bounds at every instance were obtained with inequality sets $(A B)$ and $(A B C)$.

In all of the instances Gurobi found the optimal solution within at most $12 \mathrm{~min}$. In Table 1 , for each instance we highlighted the best CPU time requirement in bold. Observe that valid inequality sets (B) and (BC) resulted in lower CPU times in majority of the instances. When the CPU times were ranked for each instance, set $(A B C)$ was among the first three in 5 out of 12 instances. In terms of the number of nodes in the branch and bound tree, on the average, set $(\mathrm{BC})$ showed the best behavior, whereas $(\mathrm{ABC})$ was the second.

In light of these observations, since the behavior of $(A B C)$ is fairly satisfactory at each instance, we decided to include all sets of valid inequalities for the rest of the computational analysis.

\subsection{A heuristic algorithm}

From our preliminary analysis with (P1), we observed that it is very difficult to solve it to optimality even with the inclusion of the valid inequalities. Thus, in this section, we introduce a simple heuristic for the problem and test its performance.

Even though there are various studies proposing heuristics for hub location problems with transportation cost objectives (for example $[15,16,21,22,24,38])$ and few for the center problem $[23,31,37]$, to the best our knowledge, there is a single study presenting a heuristic for the hub covering problem. In this study by Calik et al. [8], the authors developed a tabu search based heuristic for the incomplete hub covering problem. The main challenge in the tabu search implementation was to find feasible solutions for tight service time bounds. With this observation, we believe it will be even harder to generate feasible solutions for our problem since there are two service time bounds that have to be taken into account instead of one.

In Alumur et al. [3], an efficient formulation for the incomplete hub covering problem is introduced. The authors were able to solve the largest incomplete hub network design problems with their formulation within few seconds to optimality using the optimization software CPLEX 11.2. This observation led us to consider the solution potential of the multimodal hub covering network design problem with two service time promises. The difference of this problem from the incomplete hub covering problem introduced in Alumur et al. [3] is that there are two different service time promises, and two different types of hubs and hub links to establish.

Since it is difficult to obtain feasible solutions for the problem, initially transportation costs can be neglected and the emphasis can be made on obtaining feasible hub networks in terms of service time promises. Thus, we now define a new problem which we refer to as the covering version of (P1), where we simply remove the transportation costs from the objective function (21) and constraints (27), (32) and (33) from (P1). With the preliminary experiments by using the Turkish network data set, we observed that Gurobi is able to solve this covering version of the multimodal problem optimally within reasonable CPU times.

Note that, since only the transportation costs and the related variables are excluded, the solution from the covering version of the problem will always be feasible to the original problem (P1).
Even though the optimal covering solution is feasible to the original problem it may not be reasonable in terms of transportation costs. As long as a given origin-destination pair satisfies its service time limit the allocation decisions are insignificant since allocations do not have any effect on the objective function. Thus, if the solution from the covering version is to be proposed for the original problem an additional optimization for the allocations considering the transportation costs will be necessary. Our simple heuristic is based on this idea.

We propose the following iterative solution method as a heuristic. In the first step of the algorithm, the covering version of the problem is to be solved to optimality. Then, in the second step, the original problem is solved by fixing the hub locations and the air hub links obtained from the covering version to optimize for the allocations and the transportation costs.

We expect to obtain good quality solutions especially for tight service time bounds using our heuristic. This is because with tight service time bounds, the number of feasible hub locations and hub networks are typically limited. Thus, the optimal hub locations and the hub network for the covering version will most likely be also optimal for the original version.

In order to comment on the performance of this heuristic algorithm, we need to have optimal solutions. However, it is not possible to obtain the optimal solutions of the problem on the Turkish network data set with 81 demand centers. Thus, to test the performance of the heuristic we use the smaller data set from the Turkish network with 25 demand nodes and 8 candidate hub locations, which is also used for testing the performance of the valid inequalities in the previous section.

Initially, we solve the linear integer programming formulation of (P1) at the instances listed in Table 2 by including all sets of valid inequalities. Then, we run our heuristic algorithm for the same instances. While solving the covering version of the problem, the first phase of the heuristic, we include the valid inequality set (C) which is related to travel times only, and while optimizing the allocations, the second phase of the heuristic, we include the remaining sets of valid inequalities (A) and (B).

In Table 2, for each instance we list the CPU time requirements in seconds and the percent gap of the heuristic from the optimal solution. When listing the CPU times, the column denoted by ' $\mathrm{CPU}$ Cover' provides the CPU time requirement by Gurobi for the first phase of the heuristic where we solve the covering version of the problem and the column denoted by 'CPU Allocations' for the second phase of the heuristic where we solve for the optimal allocations. The 'CPU Total' column, on the other hand, presents the total CPU time requirement by the heuristic algorithm. The last row in Table 2 lists the average values for each column.

Note that since the covering version of the problem does not contain any variables related to transportation costs, the solutions are independent from the cost ratio $r$. So, it suffices to solve the covering version of the problem only once for each $p$ and $q$ combination. Observe from the 'CPU Cover' column of Table 2 that the CPU times corresponding to each $p$ and $q$ combination are the same and independent from $r$.

The CPU performance of the heuristic is very promising. The worst total CPU time in Table 2 is just above $12 \mathrm{~s}$, whereas the best is about $2 \mathrm{~s}$. The covering version of the problem is solved very efficiently on the average within $2 \mathrm{~s}$, whereas the allocation sub-problem is solved on the average within $3.41 \mathrm{~s}$. On the average the total CPU time requirement of the heuristic is $5.39 \mathrm{~s}$.

Our heuristic is able to find the optimal solution in 12 out of the 40 instances listed in Table 2 . The highest gap of the heuristic is $1.32 \%$ whereas the average gap is $0.22 \%$.

In order to observe the performance of the heuristic algorithm on a different data set, we generated a set of test instances from the well-known CAB data set [5]. Since not all the parameter 
Table 2

Performance of the heuristic algorithm on the 25-8 Turkish network.

\begin{tabular}{|c|c|c|c|c|c|c|}
\hline $\boldsymbol{p}$ & $\mathbf{q}$ & $\boldsymbol{r}$ & CPU cover (s) & CPU allocations (s) & CPU total (s) & Gap (\%) \\
\hline 4 & 2 & 2 & 1.53 & 0.48 & 2.01 & 0.04 \\
\hline 4 & 2 & 4 & 1.53 & 0.58 & 2.11 & 0.21 \\
\hline 4 & 2 & 6 & 1.53 & 0.62 & 2.15 & 0.43 \\
\hline 4 & 2 & 8 & 1.53 & 0.60 & 2.13 & 0.59 \\
\hline 4 & 3 & 2 & 1.98 & 0.65 & 2.63 & 0 \\
\hline 4 & 3 & 4 & 1.98 & 0.88 & 2.86 & 0 \\
\hline 4 & 3 & 6 & 1.98 & 0.67 & 2.65 & 0 \\
\hline 4 & 3 & 8 & 1.98 & 0.65 & 2.63 & 0 \\
\hline 4 & 4 & 2 & 1.79 & 1.85 & 3.64 & 0.17 \\
\hline 4 & 4 & 4 & 1.79 & 2.78 & 4.57 & 0.54 \\
\hline 4 & 4 & 6 & 1.79 & 2.95 & 4.74 & 0.93 \\
\hline 4 & 4 & 8 & 1.79 & 3.25 & 5.04 & 1.32 \\
\hline 5 & 2 & 2 & 1.88 & 1.91 & 3.79 & 0.05 \\
\hline 5 & 2 & 4 & 1.88 & 1.51 & 3.39 & 0.07 \\
\hline 5 & 2 & 6 & 1.88 & 1.42 & 3.30 & 0.07 \\
\hline 5 & 2 & 8 & 1.88 & 1.53 & 3.41 & 0.07 \\
\hline 5 & 3 & 2 & 1.58 & 1.81 & 3.39 & 0 \\
\hline 5 & 3 & 4 & 1.58 & 2.03 & 3.61 & 0 \\
\hline 5 & 3 & 6 & 1.58 & 2.37 & 3.95 & 0 \\
\hline 5 & 3 & 8 & 1.58 & 2.31 & 3.89 & 0 \\
\hline 5 & 4 & 2 & 1.81 & 2.12 & 3.93 & 0.10 \\
\hline 5 & 4 & 4 & 1.81 & 2.62 & 4.43 & 0.26 \\
\hline 5 & 4 & 6 & 1.81 & 4.53 & 6.34 & 0.43 \\
\hline 5 & 4 & 8 & 1.81 & 4.88 & 6.69 & 0.55 \\
\hline 5 & 5 & 2 & 1.83 & 4.26 & 6.09 & 0.03 \\
\hline 5 & 5 & 4 & 1.83 & 3.88 & 5.71 & 0.15 \\
\hline 5 & 5 & 6 & 1.83 & 4.15 & 5.98 & 0.31 \\
\hline 5 & 5 & 8 & 1.83 & 4.99 & 6.82 & 0.43 \\
\hline 6 & 4 & 2 & 3.77 & 2.63 & 6.40 & 0.09 \\
\hline 6 & 4 & 4 & 3.77 & 5.23 & 9.00 & 0.25 \\
\hline 6 & 4 & 6 & 3.77 & 7.05 & 10.82 & 0.42 \\
\hline 6 & 4 & 8 & 3.77 & 5.92 & 9.69 & 0.54 \\
\hline 6 & 5 & 2 & 1.95 & 2.98 & 4.93 & 0.02 \\
\hline 6 & 5 & 4 & 1.95 & 5.70 & 7.65 & 0.15 \\
\hline 6 & 5 & 6 & 1.95 & 6.54 & 8.49 & 0.31 \\
\hline 6 & 5 & 8 & 1.95 & 6.38 & 8.33 & 0.43 \\
\hline 6 & 6 & 2 & 1.70 & 5.60 & 7.30 & 0 \\
\hline 6 & 6 & 4 & 1.70 & 8.24 & 9.94 & 0 \\
\hline 6 & 6 & 6 & 1.70 & 10.52 & 12.22 & 0 \\
\hline 6 & 6 & 8 & 1.70 & 7.38 & 9.08 & 0 \\
\hline \multicolumn{3}{|c|}{ Average } & 1.98 & 3.41 & 5.39 & 0.22 \\
\hline
\end{tabular}

values are available on the $C A B$ data set, we estimated the values based on our observations from the Turkish network. There are 25 nodes in the CAB data set and we took the first 15 of them to be the candidate hub set. We took the fixed hub costs to be inversely proportional to the demand generated at the nodes so as to favor high demand generating nodes. The fixed link costs, on the other hand, are taken from Calik et al. [8]. We took the service bounds as $\beta_{1}=1000$ and $\beta_{2}=2000$ miles [10]. We assumed that the first seven nodes in the network mutually receive service in $\beta_{1}$. The other parameter values comply with the values generated for the Turkish network. Note that we generated instances on the CAB data set only for measuring the performance of the heuristic, not for analyzing the resulting hub networks. The results with varying values of $p, q$, and $r$ with the CAB data set are presented in Table 3 .

The CPU time requirement for the cover problem increases with the $C A B$ data set due to the increase on the candidate number of hub locations. Allocation problem on the other hand is solved more effectively in approximately one second. On the average the total CPU time requirement of the heuristic is less than $15 \mathrm{~s}$ with the $\mathrm{CAB}$ instances.

Our heuristic obtained optimal solutions in 12 out of the 28 instances presented in Table 3. The average gap of the heuristic is $2.36 \%$ and the maximum gap is $8.70 \%$ on these instances. At the instances with relatively higher gaps, the locations of a few hub nodes differ from the optimal solution. Observe from Table 3 that the gaps of the heuristic get higher with higher $r$ values. This is
Table 3

Performance of the heuristic algorithm with the 25-15 CAB data set.

\begin{tabular}{|c|c|c|c|c|c|c|}
\hline $\boldsymbol{p}$ & $q$ & $\boldsymbol{r}$ & CPU cover $(s)$ & CPU allocations (s) & CPU total (s) & Gap (\%) \\
\hline 4 & 2 & 2 & 7.23 & 0.53 & 7.76 & 0 \\
\hline 4 & 2 & 4 & 7.23 & 0.51 & 7.74 & 0 \\
\hline 4 & 2 & 6 & 7.23 & 0.49 & 7.72 & 0 \\
\hline 4 & 2 & 8 & 7.23 & 0.54 & 7.77 & 0 \\
\hline 4 & 3 & 2 & 2.46 & 0.58 & 3.04 & 0 \\
\hline 4 & 3 & 4 & 2.46 & 0.58 & 3.04 & 0 \\
\hline 4 & 3 & 6 & 2.46 & 0.57 & 3.03 & 0 \\
\hline 4 & 3 & 8 & 2.46 & 0.73 & 3.19 & 0 \\
\hline 4 & 4 & 2 & 2.65 & 3.03 & 5.68 & 1.56 \\
\hline 4 & 4 & 4 & 2.65 & 2.59 & 5.24 & 2.85 \\
\hline 4 & 4 & 6 & 2.65 & 2.89 & 5.54 & 5.80 \\
\hline 4 & 4 & 8 & 2.65 & 2.04 & 4.69 & 7.84 \\
\hline 5 & 2 & 2 & 39.69 & 0.66 & 40.35 & 0.36 \\
\hline 5 & 2 & 4 & 39.69 & 0.68 & 40.37 & 1.28 \\
\hline 5 & 2 & 6 & 39.69 & 0.98 & 40.67 & 1.97 \\
\hline 5 & 2 & 8 & 39.69 & 0.97 & 40.66 & 2.54 \\
\hline 5 & 3 & 2 & 17.93 & 0.79 & 18.72 & 0 \\
\hline 5 & 3 & 4 & 17.93 & 0.93 & 18.86 & 0 \\
\hline 5 & 3 & 6 & 17.93 & 1.00 & 18.93 & 0 \\
\hline 5 & 3 & 8 & 17.93 & 1.11 & 19.04 & 0 \\
\hline 5 & 4 & 2 & 15.52 & 1.22 & 16.74 & 2.23 \\
\hline 5 & 4 & 4 & 15.52 & 1.28 & 16.80 & 4.45 \\
\hline 5 & 4 & 6 & 15.52 & 1.19 & 16.71 & 6.33 \\
\hline 5 & 4 & 8 & 15.52 & 1.34 & 16.86 & 8.11 \\
\hline 5 & 5 & 2 & 8.75 & 1.78 & 10.53 & 1.92 \\
\hline 5 & 5 & 4 & 8.75 & 2.28 & 11.03 & 4.17 \\
\hline 5 & 5 & 6 & 8.75 & 2.34 & 11.09 & 6.06 \\
\hline 5 & 5 & 8 & 8.75 & 2.12 & 10.87 & 8.70 \\
\hline \multicolumn{3}{|c|}{ Average } & 13.46 & 1.28 & 14.74 & 2.36 \\
\hline
\end{tabular}

because the magnitude of the transportation costs in the objective function value is higher when the $r$ value is higher. Thus, gaps increase with increasing transportation costs.

When we compare Tables 2 and 3, we may conclude that the heuristic algorithm performed better on the Turkish network compared with the CAB data set. This may be because of the spatial distribution of the demand nodes in the $C A B$ data set. Demand nodes are more evenly distributed in the Turkish network compared with the CAB data set.

In conclusion, we believe that our heuristic algorithm obtains good quality solutions in reasonable CPU times both with the Turkish network and the $\mathrm{CAB}$ data set. We used this heuristic to solve the multimodal hub location and hub network design problem on the Turkish data set with 81 demand centers and 16 candidate hub locations. The results are provided in the next section.

\subsection{Turkish network solutions}

With an efficient heuristic at hand, we are now able to solve the problem on the original Turkish network data set with 81 demand centers and 16 candidate hub locations.

We solved the problem with the Turkish network on the instances listed in Table 4. This table also reports the CPU time requirement for the two phases of the heuristic algorithm, the total CPU time requirement for the heuristic, and the locations of the air and ground hub nodes.

The worst CPU time of the algorithm was about 13 min which was observed at the instances locating five hubs and two air hub links. On the average, the model is solved within $8 \mathrm{~min}$. We believe these CPU times are reasonable for such a strategic problem.

Observe from Table 4 that the covering version of the problem is harder to solve on sparse hub networks, whereas the allocation sub-problem becomes harder when there are more hub links. 
Table 4

Turkish network solutions.

\begin{tabular}{|c|c|c|c|c|c|c|c|}
\hline $\boldsymbol{p}$ & $q$ & $\boldsymbol{r}$ & CPU cover $(s)$ & CPU allocations (s) & CPU total (s) & Air hub locations & Ground hub locations \\
\hline 4 & 2 & 2 & 51.73 & 15.82 & 67.55 & $34,35,58$ & 7 \\
\hline 4 & 2 & 4 & 51.73 & 52.81 & 104.54 & $34,35,58$ & 7 \\
\hline 4 & 2 & 6 & 51.73 & 63.32 & 115.05 & $34,35,58$ & 7 \\
\hline 4 & 2 & 8 & 51.73 & 51.24 & 102.97 & $34,35,58$ & 7 \\
\hline 4 & 3 & 2 & 15.65 & 53.29 & 68.94 & $34,35,58$ & 7 \\
\hline 4 & 3 & 4 & 15.65 & 66.04 & 81.69 & $34,35,58$ & 7 \\
\hline 4 & 3 & 6 & 15.65 & 76.03 & 91.68 & $34,35,58$ & 7 \\
\hline 4 & 3 & 8 & 15.65 & 73.19 & 88.84 & $34,35,58$ & 7 \\
\hline 4 & 4 & 2 & 13.64 & 84.65 & 98.29 & $7,34,35,58$ & - \\
\hline 4 & 4 & 4 & 13.64 & 118.86 & 132.50 & $7,34,35,58$ & - \\
\hline 4 & 4 & 6 & 13.64 & 87.66 & 101.30 & $7,34,35,58$ & - \\
\hline 4 & 4 & 8 & 13.64 & 93.22 & 106.86 & $7,34,35,58$ & - \\
\hline 5 & 2 & 2 & 632.03 & 125.54 & 757.57 & $34,35,58$ & 7,16 \\
\hline 5 & 2 & 4 & 632.03 & 159.48 & 791.51 & $34,35,58$ & 7,16 \\
\hline 5 & 2 & 6 & 632.03 & 162.49 & 794.52 & $34,35,58$ & 7,16 \\
\hline 5 & 2 & 8 & 632.03 & 136.81 & 768.84 & $34,35,58$ & 7,16 \\
\hline 5 & 3 & 2 & 249.73 & 123.09 & 372.82 & $34,35,58$ & 7,16 \\
\hline 5 & 3 & 4 & 249.73 & 137.37 & 387.10 & $34,35,58$ & 7,16 \\
\hline 5 & 3 & 6 & 249.73 & 135.31 & 385.04 & $34,35,58$ & 7,16 \\
\hline 5 & 3 & 8 & 249.73 & 147.00 & 396.73 & $34,35,58$ & 7,16 \\
\hline 5 & 4 & 2 & 82.34 & 114.94 & 197.28 & $16,25,34,35$ & 42 \\
\hline 5 & 4 & 4 & 82.34 & 119.49 & 201.83 & $16,25,34,35$ & 42 \\
\hline 5 & 4 & 6 & 82.34 & 119.08 & 201.42 & $16,25,34,35$ & 42 \\
\hline 5 & 4 & 8 & 82.34 & 107.78 & 190.12 & $16,25,34,35$ & 42 \\
\hline 5 & 5 & 2 & 105.96 & 195.50 & 301.46 & $16,25,34,35$ & 42 \\
\hline 5 & 5 & 4 & 105.96 & 219.00 & 324.96 & $16,25,34,35$ & 42 \\
\hline 5 & 5 & 6 & 105.96 & 174.33 & 280.29 & $16,25,34,35$ & 42 \\
\hline 5 & 5 & 8 & 105.96 & 211.26 & 317.22 & $16,25,34,35$ & 42 \\
\hline \multicolumn{3}{|c|}{ Average } & $\begin{array}{l}361.95 \\
(\approx 6.03 \mathrm{~min})\end{array}$ & $\begin{array}{l}119.41 \\
(\approx 1.99 \mathrm{~min})\end{array}$ & $\begin{array}{l}481.36 \\
(\approx 8.02 \mathrm{~min})\end{array}$ & & \\
\hline
\end{tabular}

These outcomes are expected and can be explained as follows. With less number of air hub links to establish, there are fewer feasible solutions (especially with tight service time bounds) thus, it takes more time to solve the covering problem. On the contrary, when there are more hub links, there are more possible routing alternatives while solving the allocation sub-problem and thus it takes more time.

Fig. 2(a)-(f) depicts some examples of resulting hub network solutions from the Turkish network with $r=6$.

Air and ground hubs and hub links are explicitly indicated in Fig. 2 (air hub links denoted by a double line and ground hub links by a single line). For the sake of simplicity, we do not show the allocations of the demand nodes to the hub nodes in these figures.

In our results with the Turkish network data set, there is an air hub that is usually located in the eastern part of Turkey which is connected to the hub network by an air hub link. Most of the demand centers in the eastern part of the region are allocated to this hub. In the solutions, there is a hub located at the demand center Istanbul (34), since Istanbul generates the highest amount of flow. Again, there is usually a hub located at Izmir (35), which is the third highest demand generating node, serving the western part of the region. In general, the locations of the hub nodes were insensitive to the number of hub links to establish. Observe from Fig. 2 that when the number of air hub links to be located is increased by one, generally a new air hub link is added to the hub network.

In the Turkish network solutions, once the feasibility of the hub network is ensured in terms of service time promises, additional hub nodes are established in the western part of the region. This is expected since the demand is more or less concentrated on the western part of Turkey. The most industrialized region of Turkey is the north-western part where Istanbul is located in addition to some other most industrialized cities. Thus, when we locate five hubs an additional hub is located in this north-western region at Bursa (16). Note that the fixed costs of operating hub links are dependent on the distances. Thus, additional air hub links are operated between relatively closer hubs. From the analysis on the number of air hub links, the decision maker can observe the trade-off between transportation costs and operational costs.

Observe from Fig. 2 that none of the solutions employ complete hub networks. The results show that there is no need to establish a direct connection between every pair of hub nodes in order to ensure low-cost service within the given service time bounds.

\section{Conclusion}

In this paper, we introduced the multimodal hub location and hub network design problem to the literature. The model includes various observations from real life hub networks. We relaxed the assumption of building complete hub networks, considered transportation costs and travel times simultaneously, offered different types of service time promises, and considered the choice of different modes of transportation for hubs and hub links. To the best of our knowledge, there is not any study in the hub location literature including all of these stated observations from real life hub networks.

We provided a linear mixed integer programming formulation for the most general case of the multimodal hub location and hub network design problem. We additionally proposed a more compact formulation with two types of hubs and hub links and two different service time parameters. We then proposed different sets of effective valid inequalities, and a simple and efficient heuristic algorithm for this special case of the problem. Detailed computational analysis is presented using the Turkish network data set.

The proposed multimodal hub location and hub network design formulation inherently includes various hub location 
a

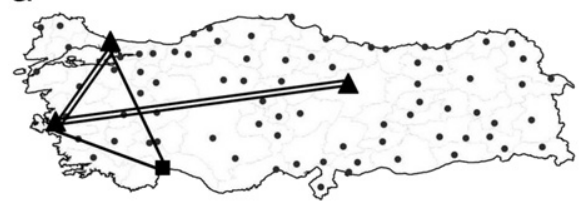

C

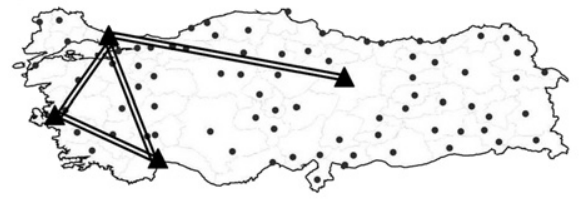

e

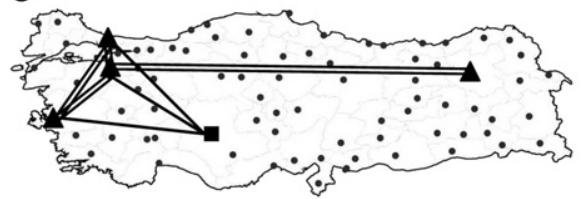

b

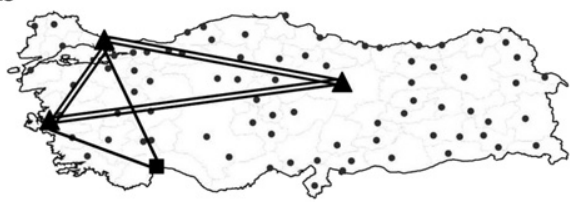

d

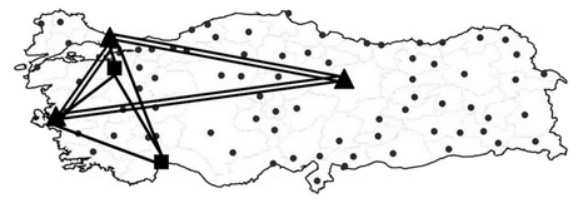

f

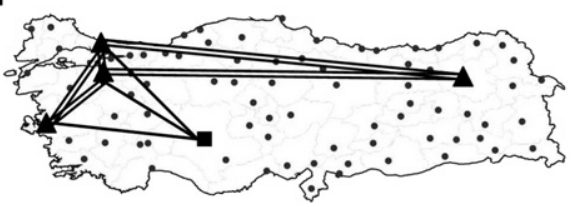

Fig. 2. Resulting hub networks with the Turkish network data set: (a) $p=4, q=2$, (b) $p=4, q=3$, (c) $p=4, q=4$, (d) $p=5, q=3$, (e) $p=5$, $q=4$, and (f) $p=5, q=5$.

models and it is a very comprehensive and a generic model. We utilized this property and introduced additional problems with respective mathematical models to the literature.

An extension to the multimodal hub location and hub network design problem is to include capacity restrictions. The proposed model is readily extendible to include capacity constraints. The capacity constraints can be on the amount of flow processed at the hubs as well as on the available number of trucks or airplanes to employ on each hub link to be established. Naturally, the problem will be harder to solve to optimality with the addition of these capacity restrictions. Thus, there will be a need to develop efficient exact solution algorithms or heuristics for the multimodal hub location and hub network design problem in the future. One other possible future research direction which will enhance the mathematical model is to include pricing issues and market competition in multimodal hub location and hub network design.

\section{Appendix}

We provide the proofs of Propositions 1-3.

Proof of Proposition 1. First we prove the validity of inequality (A.1). Consider the inequality (A.1) associated with nodes $i, j \in H: i \neq j$. There are four cases depending on the values of $X_{i i}$ and $X_{j j}$ :

Case 1: $X_{i i}=1$ and $X_{j j}=1$. Then, both of the nodes $i$ and $j$ are hubs and inequality (A.1) reduces to $C_{i j} \geq \alpha_{c}^{g} C_{i j}^{g}$. Since triangle inequality holds and $\alpha_{c}^{g} c_{i j}^{g} \leq \alpha_{c}^{a} c_{i j}^{a} \forall i, j \in N$ by assumption, the unit transportation cost from hub $i$ to hub $j$ is at least $\alpha_{c}^{g} c_{i j}^{g}$, and (A.1) is valid.

Case 2: $X_{i i}=1$ and $X_{j j}=0$. If node $i$ is a hub and node $j$ is not a hub, then the inequality (A.1) simplifies to $C_{i j} \geq \lambda_{i j}^{1}$. It is then valid by the definition of $\lambda_{i j}^{1}$.

Case 3: $X_{i i}=0$ and $X_{j j}=1$. If node $j$ is a hub and node $i$ is not a hub, then by the definition of $\lambda_{i j}^{2}$ we know that $C_{i j} \geq \lambda_{i j}^{2}$. If $\min \left\{\lambda_{i j}^{3}-\lambda_{i j}^{1}, \lambda_{i j}^{2}-\alpha_{c}^{g} C_{i j}^{g}\right\}=\lambda_{i j}^{2}-\alpha_{c}^{g} C_{i j}^{g}$, then inequality (A.1) simplifies to $C_{i j} \geq \lambda_{i j}^{2}$ and it is satisfied. If $\min \left\{\lambda_{i j}^{3}-\lambda_{i j}^{1}, \lambda_{i j}^{2}-\alpha_{c}^{g} c_{i j}^{g}\right\}=\lambda_{i j}^{3}-\lambda_{i j}^{1}$, then $\lambda_{i j}^{2}-\alpha_{c}^{g} c_{i j}^{g}+\alpha_{c}^{g} c_{i j}^{g} \geq \lambda_{i j}^{3}-\lambda_{i j}^{1}+\alpha_{c}^{g} c_{i j}^{g}$ and $C_{i j} \geq \lambda_{i j}^{2} \geq \alpha_{c}^{g} c_{i j}^{g}+\lambda_{i j}^{3}-\lambda_{i j}^{1} \quad$ and inequality (A.1) is valid.
Case 4: $X_{i i}=0$ and $X_{j j}=0$. If neither node $i$ nor node $j$ is a hub, then by the definition of $\lambda_{i j}^{3}, C_{i j} \geq \lambda_{i j}^{3}$. If $\min \left\{\lambda_{i j}^{3}-\lambda_{i j}^{1}, \lambda_{i j}^{2}-\alpha_{c}^{g} C_{i j}^{g}\right\}=$ $\lambda_{i j}^{3}-\lambda_{i j}^{1}$, then inequality (A.1) simplifies to $C_{i j} \geq \lambda_{i j}^{3}$ and it is satisfied. If $\min \left\{\lambda_{i j}^{3}-\lambda_{i j}^{1}, \lambda_{i j}^{2}-\alpha_{c}^{g} c_{i j}^{g}\right\}=\lambda_{i j}^{2}-\alpha_{c}^{g} c_{i j}^{g}$, then inequality (A.1) reduces to $C_{i j} \geq \lambda_{i j}^{1}+\lambda_{i j}^{2}-\alpha_{c}^{g} c_{i j}^{g}$. Since, $\lambda_{i j}^{3}-\lambda_{i j}^{1} \geq \lambda_{i j}^{2}-\alpha_{c}^{g} C_{i j}^{g}$, and $\lambda_{i j}^{3} \geq \lambda_{i j}^{1}$ $+\lambda_{i j}^{2}-\alpha_{c}^{g} c_{i j}^{g}$, then $C_{i j} \geq \lambda_{i j}^{3} \geq \lambda_{i j}^{1}+\lambda_{i j}^{2}-\alpha_{c}^{g} c_{i j}^{g}$ and inequality (A.1) is valid.

Since inequality (A.1) is satisfied in all of the four possible cases, we conclude that inequality (A.1) is valid.

We now prove the validity of inequality (A.2). Similarly, there are four cases depending on the values of $X_{i i}$ and $X_{j j}$ :

Case 1: $X_{i i}=0$ and $X_{j j}=0$. If neither of the nodes $i$ and $j$ are hub, the inequality (A.2) reduces to $C_{i j} \geq \lambda_{i j}^{3}$ and it is satisfied by the definition of $\lambda_{i j}^{3}$.

Case 2: $X_{i i}=0$ and $X_{j j}=1$. If node $i$ is not a hub and node $j$ is a hub, then the inequality simplifies to $C_{i j} \geq \lambda_{i j}^{2}$ and it is again valid by definition.

Case 3: $X_{i i}=1$ and $X_{j j}=0$. If node $i$ is a hub and node $j$ is not a hub, then we know that $C_{i j} \geq \lambda_{i j}^{1}$ by definition. If $\min \left\{\lambda_{i j}^{1}-\lambda_{i j}^{3}, \alpha_{c}^{g} c_{i j}^{g}-\lambda_{i j}^{2}\right\}=\lambda_{i j}^{1}-\lambda_{i j}^{3}$, the inequality (A.2) simplifies to $C_{i j} \geq \lambda_{i j}^{1}$ and it is valid. If $\min \left\{\lambda_{i j}^{1}-\lambda_{i j}^{3}, \alpha_{c}^{g} c_{i j}^{g}-\lambda_{i j}^{2}\right\}=\alpha_{c}^{g} c_{i j}^{g}-\lambda_{i j}^{2}$, then $\lambda_{i j}^{1}-\lambda_{i j}^{3} \geq \alpha_{c} c_{i j}^{g}-\lambda_{i j}^{2}$ and $C_{i j} \geq \lambda_{i j}^{1} \geq \lambda_{i j}^{3}+\alpha_{c} c_{i j}^{g}-\lambda_{i j}^{2}$. Hence, inequality (A.2) is valid.

Case 4: $X_{i i}=1$ and $X_{j j}=1$. Then, inequality (A.2) reduces to $C_{i j} \geq \lambda_{i j}^{2}+\min \left\{\lambda_{i j}^{1}-\lambda_{i j}^{3}, \alpha_{c}^{g} c_{i j}^{g}-\lambda_{i j}^{2}\right\}$. If both of the nodes $i$ and $j$ are hubs, we know that $C_{i j} \geq \alpha_{c}^{g} c_{i j}^{g}$ by assumption. If $\min \left\{\lambda_{i j}^{1}-\lambda_{i j}^{3}\right.$, $\left.\alpha_{c}^{g} c_{i j}^{g}-\lambda_{i j}^{2}\right\}=\alpha_{c}^{g} c_{i j}^{g}-\lambda_{i j}^{2}$, then inequality reduces to $C_{i j} \geq \alpha_{c}^{g} c_{i j}^{g}$ and it is satisfied. If $\min \left\{\lambda_{i j}^{1}-\lambda_{i j}^{3}, \alpha_{c}^{g} c_{i j}^{g}-\lambda_{i j}^{2}\right\}=\lambda_{i j}^{1}-\lambda_{i j}^{3}$, then $\alpha_{c}^{g} c_{i j}^{g}-\lambda_{i j}^{2} \geq \lambda_{i j}^{1}-$ $\lambda_{i j}^{3}$. So, $C_{i j} \geq \alpha_{c}^{g} c_{i j}^{g} \geq \lambda_{i j}^{2}+\lambda_{i j}^{1}-\lambda_{i j}^{3}$ and the inequality is satisfied.

Inequality (A.2) is satisfied in all of the four possible cases. So, we conclude that inequality (A.2) is valid.

If node $i$ is a hub, inequality (A.3) simplifies to $C_{i j} \geq \lambda_{i j}^{1}$ and it is valid by definition since $j \in N \backslash H$. If $i$ is not a hub it reduces to $C_{i j} \geq \lambda_{i j}^{3}$ and it is valid again by definition. Similarly, inequality (A.4) for $i \in N \backslash H$, reduces to $C_{i j} \geq \lambda_{i j}^{2}$ when $j$ is a hub, and to $C_{i j} \geq \lambda_{i j}^{3}$ when $j$ is not a hub and valid for both of the cases. For inequality (A.5), since $i, j \in N \backslash H, C_{i j} \geq \lambda_{i j}^{3}$ by definition.

Hence, inequalities (A.1)-(A.5) are valid. 
Proof of Proposition 2. Let $i \in H$ and $j \in N \backslash\{i\}$. If $X_{i i}=1$, then $\sum_{h \in H \backslash i\}} X_{i h}=0$ and inequality (B.1) reduces to $C_{i j} \geq \min _{k \in H}$ $\left(\alpha_{c}^{g} c_{i k}^{g}+c_{k j}^{g}\right)$. Independent of whether node $j$ is a hub or not, since node $k$ may be equal to node $j, \min _{k \in H}\left(\alpha_{c}^{g} c_{i k}^{g}+c_{k j}^{g}\right)$ provides a lower bound on the unit transportation cost from node $i$ to node $j$, by triangle inequality and by the assumption that $\alpha_{c}^{g} c_{i j}^{g} \leq c_{i j}^{a} \forall i$, $j \in N$. So, inequality (B.1) is satisfied. If $X_{i i}=0$, then node $i$ must be allocated to a hub, say $h$, such that $X_{i h}=1$ (node $h$ is allowed to be the same node as $j$ ), then the unit transportation cost from hub $h$ to node $j$ is at least $\min _{k \in H \backslash\{i\}}\left(\alpha_{c}^{g} c_{h k}^{g}+c_{k j}^{g}\right)$. So, $C_{i j} \geq c_{i h}^{g}+\min _{k \in H \backslash i i\}}$ $\left(\alpha_{c}^{g} c_{h k}^{g}+c_{k j}^{g}\right)$. Hence, inequality (B.1) is valid.

For (B.2), $i \in N \backslash H$ and $j \in N \backslash\{i\}$, and thus $X_{i h}=1$ for some hub $h$. Then from the above argument of the proof of inequality (B.1) the unit transportation cost from hub $h$ to node $j$ is at least $\min _{k \in H \backslash i i}$ $\left(\alpha_{c}^{g} c_{h k}^{g}+c_{k j}^{g}\right)$. So, $C_{i j} \geq \sum_{h \in H}\left(c_{i h}^{g}+\min _{k \in H}\left(\alpha_{c}^{g} c_{h k}^{g}+c_{k j}^{g}\right)\right) X_{i h}$ and (B.2) is valid.

For $i \in N$ and $j \in H \backslash\{i\}$, if $X_{j j}=1$, then $\sum_{h \in H \backslash j\}} X_{j h}=0$ and it takes at least $\min _{k \in H}\left(c_{i k}^{g}+\alpha_{c}^{g} c_{k j}^{g}\right)$ units of transportation cost to travel from node $i$ to node $j$. Hence, $C_{i j} \geq \min _{k \in H}\left(c_{i k}^{g}+\alpha_{c}^{g} c_{k j}^{g}\right)$ and inequality (B.3) is satisfied. If $X_{j j}=0$, then it is allocated to a hub, say $h$, and the unit transportation cost from node $i$ to hub $h$ is at least $\min _{k \in H \backslash j\}}\left(c_{i k}^{g}+\alpha_{c}^{g} c_{k h}^{g}\right)$. Hence, $C_{i j} \geq \min _{k \in H \backslash j\}}\left(c_{i k}^{g}+\alpha_{c}^{g} c_{k h}^{g}\right)+c_{h j}^{g}$ and inequality (B.3) is valid. Similarly for (B.4), since $X_{j j}=0$, $C_{i j} \geq \sum_{h \in H}\left(\min _{k \in H}\left(c_{i k}^{g}+\alpha_{c}^{g} c_{k h}^{g}\right)+c_{h j}^{g}\right) X_{j h}$ from the same argument. Hence, (B.4) is also valid.

So, inequalities (B.1)-(B.4) are valid.

Proof of Proposition 3. The inequalities (C.1) and (C.2) are very similar in structure to the inequalities (A.1) and (A.2). The only difference is that the unit transportation cost values are appropriately replaced with travel times. Thus, the proofs of the validity of the inequalities (C.1) and (C.2) follow from the proofs of the valid inequalities (A.1) and (A.2) from Proposition 1. Similarly, the inequalities (C.3) and (C.4) are similar in structure with the inequalities (B.1) and (B.3), and the validity of these inequalities follow from the proofs of the valid inequalities (B.1) and (B.3) from Proposition 2.

\section{References}

[1] Alumur S, Kara BY. Network hub location problems: the state of the art European Journal of Operational Research 2008;190:1-21.

[2] Alumur S, Kara BY. A hub covering network design problem for cargo applications in Turkey. Journal of the Operational Research Society 2009;60:1349-59.

[3] Alumur SA, Kara BY, Karasan OE. The design of single allocation incomplete hub networks. Transportation Research Part B 2009;43:936-51.

[4] Arnold P, Peeters D, Thomas I. Modelling a rail/road intermodal transportation system. Transportation Research Part E 2004;40:255-70.

[5] Beasley JE. OR-library: hub location. 〈http://people.brunel.ac.uk/ mastjjb/ jeb/orlib/phubinfo.html $>$; 1990. Accessed 15.03.2010. Site last updated February 2008.

[6] Bontekoning YM, Macharis C, Trip JJ. Is a new applied transportation research field emerging? A review of intermodal rail-truck freight transport literature Transportation Research Part A 2004;38:1-34.

[7] Boysen N, Fliedner M. Cross dock scheduling: classification, literature review and research agenda. Omega 2010;38:413-22.

[8] Calik H, Alumur SA, Kara BY, Karasan OE. A tabu-search based heuristic for the hub covering problem over incomplete hub networks. Computers and Operations Research 2009;36:3088-96.

[9] Campbell JF. Integer programming formulations of discrete hub location problems. European Journal of Operational Research 1994;72:387-405.

[10] Campbell JF. Hub location for time definite transportation. Computers and Operations Research 2009;36:3107-16.

[11] Campbell JF, Ernst AT, Krishnamoorthy M. Hub Location Problems. In: Drezner Z, Hamacher H, editors. Facility location: applications and theory. Berlin: Springer-Verlag; 2002.

[12] Campbell JF, Ernst AT, Krishnamoorthy M. Hub arc location problems: part I-introduction and results. Management Science 2005;51:1540-55.
[13] Campbell JF, Ernst AT, Krishnamoorthy M. Hub arc location problems: part II-formulations and optimal algorithms. Management Science 2005;51: 1556-71.

[14] Campbell JF, Stiehr G, Ernst AT, Krishnamoorthy M. Solving hub arc location problems on a cluster of workstations. Parallel Computing 2003;29: 555-74.

[15] Canovas L, Garcia S, Marin A. Solving the uncapacitated multiple allocation hub location problem by means of a dual-ascent technique. European Journal of Operational Research 2007;179:990-1007.

[16] Chen JF. A hybrid heuristic for the uncapacitated single allocation hub location problem. Omega 2007;35:211-20.

[17] Contreras I, Fernandez E, Marin A. Tight bounds from a path based formulation for the tree of hub location problem. Computers and Operations Research 2009;36:3117-27.

[18] Contreras I, Fernandez E, Marin A. The tree of hubs location problem. European Journal of Operational Research 2010;202:390-400.

[19] Costa AM, Franca PM, Filho CL. Two-level network design with intermediate facilities: an application to electrical distribution systems. Omega 2011;39: 3-13.

[20] Crainic TG, Kim KH. Intermodal transportation. In: Barnhart C, Laporte G, editors. Transportation. Amsterdam: North Holland; 2007.

[21] Cunha CB, Silva MR. A genetic algorithm for the problem of configuring a hub-and-spoke network for a LTL trucking company in Brazil. European Journal of Operational Research 2007;179:747-58.

[22] Ebery J, Krishnamoorthy M, Ernst A, Boland N. The capacitated multiple allocation hub location problem: formulations and algorithms. European Journal of Operational Research 2000;120:614-31.

[23] Ernst AT, Hamacher H, Jiang H, Krishnamoorthy M, Woeginger G. Uncapacitated single and multi allocation $p$-hub center problems. Computers and Operations Research 2009;36:2230-41.

[24] Ernst AT, Krishnamoorthy M. Efficient algorithms for the uncapacitated single allocation p-hub median problem. Location Science 1996;4:139-54.

[25] Groothedde B, Ruijgrok C, Tavasszy L. Towards collaborative, intermodal hub networks: a case study in the fast moving consumer goods market. Transportation Research Part E 2005;41:567-83.

[26] Ishfaq R, Sox CR. Intermodal logistics: the interplay of financial, operational and service issues. Transportation Research Part E 2010;46:926-49.

[27] Ishfaq R, Sox CR. Hub location-allocation in intermodal logistic networks. European Journal of Operational Research 2011;210:213-30.

[28] Limbourg S, Jourquin B. Optimal rail-road container terminal locations on the European network. Transportation Research Part E 2009;45:551-63.

[29] Ma H, Miao Z, Lim A, Rodriguez B. Crossdocking distribution networks with setup cost and time window constraint. Omega 2011;39:64-72.

[30] Meng Q, Wang X. Intermodal hub-and-spoke network design: incorporating multiple stakeholders and multi-type containers. Transportation Research Part B 2011;45:724-42.

[31] Meyer T, Ernst AT, Krishnamoorthy M. A 2-phase algorithm for solving the single allocation p-hub center problem. Computers and Operations Research 2009;36:3143-51.

[32] Nickel S, Schobel A, Sonneborn T. Hub Location problems in urban traffic networks. In: Niittymaki J, Pursula M, editors. Mathematics methods and optimization in transportation systemsKluwer Academic Publishers; 2001. p. 1-12. [Chapter 1]

[33] O'Kelly ME. The location of interacting hub facilities. Transportation Science 1986;20:92-105

[34] O'Kelly ME. A quadratic integer program for the location of interacting hub facilities. European Journal of Operational Research 1987;32:393-404.

[35] O'Kelly ME, Lao Y. Mode choice in a hub-and-spoke network: a zero-one linear programming approach. Geographical Analysis 1991;23:283-397.

[36] O'Kelly ME, Miller HJ. The hub network design problem: a review and synthesis. Journal of Transport Geography 1994;2:31-40.

[37] Pamuk FS, Sepil C. A solution to the hub center problem via a singlerelocation algorithm with tabu search. IIE Transactions 2001;33:399-411.

[38] Pirkul H, Schilling DA. An efficient procedure for designing single allocation hub and spoke systems. Management Science 1998;44:235-42.

[39] Podnar H, Skorin-Kapov J, Skorin-Kapov D. Network cost minimization using threshold-based discounting. European Journal of Operational Research 2002;137:371-86.

[40] Racunica I, Wynter L. Optimal location of intermodal freight hubs. Transportation Research Part B 2005;39:453-77.

[41] Tan PZ, Kara BY. A hub covering model for cargo delivery systems. Networks 2007;49:28-39.

[42] Tyan JC, Wang F-K, Du TC. An evaluation of freight consolidation policies in global third party logistics. Omega 2003;31:55-62.

[43] Van Belle J, Valckenaers P, Cattrysse D. Cross-docking: state of the art. Omega 2013;41.

[44] Yaman H, Kara BY, Tansel BC. The latest arrival hub location problem for cargo delivery systems with stopovers. Transportation Research Part B 2007;41:906-19.

[45] Yaman H, Karasan OE, Kara BY. Release time scheduling in cargo delivery. Operations Research, accepted for publication.

[46] Yoon MG, Current J. The hub location and network design problem with fixed and variable arc costs: formulation and dual-based solution heuristic. Journal of the Operational Research Society 2008;59:80-9. 